OPEN ACCESS

Edited by:

Weiwen Zhang,

Tianjin University, China

Reviewed by:

Iris Maldener,

University of Tübingen, Germany

Shigeki Ehira,

Tokyo Metropolitan University, Japan

Patrick Jean-Adrien Videau,

Southern Oregon University,

United States

*Correspondence:

Xiaoli Zeng

zengx।@ihb.ac.cn

Specialty section:

This article was submitted to Microbial Physiology and Metabolism,

a section of the journal

Frontiers in Microbiology

Received: 12 October 2021 Accepted: 09 November 2021

Published: 03 December 2021

Citation:

Huang $M$, Zhang $J-Y$, Zeng $X$ and

Zhang C-C (2021) c-di-GMP

Homeostasis Is Critical for

Heterocyst Development in

Anabaena sp. PCC 7120.

Front. Microbiol. 12:793336.

doi: 10.3389/fmicb.2021.793336

\section{c-di-GMP Homeostasis Is Critical for Heterocyst Development in Anabaena sp. PCC 7120}

\author{
Min Huang ${ }^{1,2}$, Ju-Yuan Zhang ${ }^{1}$, Xiaoli Zeng ${ }^{1 *}$ and Cheng-Cai Zhang ${ }^{1,2,3,4}$ \\ 'State Key Laboratory of Freshwater Ecology and Biotechnology, Key Laboratory of Algal Biology, Institute of \\ Hydrobiology, Chinese Academy of Sciences, Wuhan, China, ${ }^{2}$ College of Advanced Agricultural Sciences, University of \\ Chinese Academy of Sciences, Beijing, China, ${ }^{3} / n$ stitut AMU-WUT, Aix-Marseille University and Wuhan University of \\ Technology, Wuhan, China, ${ }^{4}$ Innovation Academy for Seed Design, Chinese Academy of Sciences, Beijing, China
}

c-di-GMP is a ubiquitous bacterial signal regulating various physiological process. Anabaena PCC 7120 (Anabaena) is a filamentous cyanobacterium able to form regularlyspaced heterocysts for nitrogen fixation, in response to combined-nitrogen deprivation in $24 \mathrm{~h}$. Anabaena possesses 16 genes encoding proteins for c-di-GMP metabolism, and their functions are poorly characterized, except all2874 (cdgS) whose deletion causes a decrease in heterocyst frequency $48 \mathrm{~h}$ after nitrogen starvation. We demonstrated here that c-di-GMP levels increased significantly in Anabaena after combined-nitrogen starvation. By inactivating each of the 16 genes, we found that the deletion of all1175 $(\mathrm{cdgSH})$ led to an increase of heterocyst frequency $24 \mathrm{~h}$ after nitrogen stepdown. A double mutant $\Delta c d g S H \Delta c d g S$ had an additive effect over the single mutants in regulating heterocyst frequency, indicating that the two genes acted at different time points for heterocyst spacing. Biochemical and genetic data further showed that the functions of $\mathrm{CdgSH}$ and CdgS in the setup or maintenance of heterocyst frequency depended on their opposing effects on the intracellular levels of c-di-GMP. Finally, we demonstrated that heterocyst differentiation was completely inhibited when c-di-GMP levels became too high or too low. Together, these results indicate that the homeostasis of c-di-GMP level is important for heterocyst differentiation in Anabaena.

Keywords: cyanobacteria, c-di-GMP, nitrogen fixation, signal transduction, homeostasis

\section{INTRODUCTION}

Cyclic nucleotides act as second messengers in all domains of life (Tschowri et al., 2014). One of them, bis-(3'-5')-cyclic dimeric GMP (c-di-GMP), has emerged as one of the most common and important secondary messengers in bacteria (Romling et al., 2013). It was first discovered in Gluconacetobacter xylinus about 30 years ago, as a positive allosteric regulator of cellulose synthase (Ross et al., 1987). Subsequent studies have revealed that c-di-GMP regulates a variety of important cellular processes, including biofilm formation, motility, virulence, cell differentiation, cell cycle and mechanical sensing (Tischler and Camilli, 2004; Kulesekara et al., 2006; Hengge, 2009; Boehm et al., 2010; Romling et al., 2013; Tschowri et al., 2014; Lori et al., 2015; Hug et al., 2017; Xu et al., 2019; Del Medico et al., 2020; Snyder et al., 2020). Such regulations may 
take place at the transcriptional, post-transcriptional, or posttranslational levels by interacting with different types of effectors (Romling et al., 2013; Chou and Galperin, 2016). Several classes of c-di-GMP receptors, such as PilZ domain-containing receptors, I site receptors, inactive EAL domain receptors and riboswitches, can be identified by sequence alignment, since they usually share a conserved c-di-GMP binding motif (Romling et al., 2013; Chou and Galperin, 2016). However, other newly discovered receptors are being identified, which have no common features in protein sequences (Hickman and Harwood, 2008; Burdette et al., 2011; Tschowri et al., 2014; Lori et al., 2015; Xu et al., 2019; Gallagher et al., 2020).

The intracellular level of c-di-GMP is attributed to two opposing enzymatic activities: diguanylate cyclase (DGC) and c-di-GMP-specific phosphodiesterase (PDE). DGCs contain the conserved GG(D/E)EF motif and can synthesize one molecule of c-di-GMP using two GTPs as substrate (Chan et al., 2004; Paul et al., 2007). PDEs harbor either the EAL or the HD-GYP domain and can hydrolyze c-di-GMP into 5'-phosphoguanylyl$\left(3^{\prime}-5^{\prime}\right)$-guanosine $(\mathrm{pGpG})$ or further down to GMP (Schmidt et al., 2005; Christen et al., 2006). GG(D/E)EF, EAL or HD-GYP domains may co-occur in multi-domain proteins, together with other diverse regulatory domains, including REC (Receiver), PAS (Per-ARNT-Sim), GAF (cGMP phosphodiesterase/adenylate cyclase/FhlA), FHA (fork head-associated), and CHASE (cyclase/ histidine kinases-associated sensing extracellular). These regulatory domains control the synthesis or degradation of c-di-GMP in response to specific environmental cues, such as oxygen, light, quorum sensing signals, aromatic hydrocarbons, phosphorylation and proton flux (Paul et al., 2004; Barends et al., 2009; Tuckerman et al., 2009; Savakis et al., 2012; MartinMoldes et al., 2016; Hug et al., 2017; Yang et al., 2017). Bioinformatics analysis of completed cyanobacterial genomes indicate that most of them contain multiple genes related to c-di-GMP turnover (Romling et al., 2013). This observation suggests that, while c-di-GMP has important functions in cyanobacteria, its exact roles remain poorly characterized. In the unicellular cyanobacterium Synechocystis sp. PCC 6803, the intracellular level of c-di-GMP regulates phototaxis (Savakis et al., 2012). In Thermosynechococcus vulcanus, the intracellular c-di-GMP level is controlled by a light-quality sensitive input system formed by three cyanobacteriochromes and regulates formation of cell aggregation (Enomoto et al., 2014, 2015).

When grown under diazotrophic conditions, the filamentous cyanobacterium Anabaena/Nostoc PCC 7120 (Anabaena) has two types of cells, vegetative cells and heterocysts. Vegetative cells can divide and perform photosynthesis, whereas heterocysts are terminally differentiated, nitrogen-fixing cells (Wolk et al., 1994; Kumar et al., 2010; Muro-Pastor and Hess, 2012; Herrero and Flores, 2019). Heterocysts represent 5-10\% of all cells along the filaments (Wolk et al., 1994; Kumar et al., 2010; Muro-Pastor and Hess, 2012; Herrero and Flores, 2019), and are formed in response to deprivation of combined nitrogen. Single heterocysts are semi-regularly spaced along the filaments, and the existence of the two cell types allows Anabaena filaments to perform simultaneously two incompatible activities, oxygenlabile nitrogen fixation and oxygen-evolving photosynthesis.
Heterocysts supply vegetative cells with fixed nitrogen, and in return receive fixed carbon from vegetative cells (Wolk et al., 1994; Kumar et al., 2010; Muro-Pastor and Hess, 2012; Herrero and Flores, 2019). Heterocyst development and pattern formation are complex and highly coordinated processes, during which several signals and a large number of positive or negative regulators are involved (Zhang et al., 2006). The signals include peptides derived from PatS, PatX, HetN, serving as inhibitors for formation and maintenance of heterocyst pattern (Yoon and Golden, 1998; Callahan and Buikema, 2001; Elhai and Khudyakov, 2018), 2-oxoglutarate whose accumulation acts as a nitrogen starvation signal and a trigger for heterocyst development (Laurent et al., 2005), and ppGpp required for heterocyst differentiation (Zhang et al., 2013).

Anabaena possesses 16 genes encoding proteins with $\mathrm{GG}(\mathrm{D} / \mathrm{E})$ EF motif or EAL/HD-GYP motif; however, the physiological functions of c-di-GMP have not been well explored. A report by Neunuebel and Golden showed that the inactivation of all2874 (cdgS: c-di-GMP synthetase) encoding a diguanylate cyclase caused a significant reduction in heterocyst frequency at the maintenance steps (Neunuebel and Golden, 2008). In this mutant, the heterocyst frequency was similar to the wild type at the initial formation step, but over the next 4 days, it was decreased dramatically as compared with wild type. The intervals between heterocysts increased to as many as 200 vegetative cells, compared to less than 25 vegetative cells for the control (Neunuebel and Golden, 2008). The relationship between intracellular c-di-GMP levels and heterocysts development, and the underlying mechanism, are still unclear.

Here, we report that c-di-GMP plays a critical role in regulating heterocyst development. Our data show that c-di-GMP levels in Anabaena accumulate transiently after nitrogen starvation. By combining genetic and biochemical analyses, we established the relationship between c-di-GMP levels and heterocysts frequency under the control of two genes, all1175 (cdgSH: c-di-GMP synthetase and hydrolase) and all2874 (cdgS). We further demonstrated that the homeostasis of c-di-GMP is important for heterocyst differentiation in Anabaena. This study paves the way for characterizing the signalling function of c-di-GMP in this cyanobacterium, a model for prokaryotic development.

\section{MATERIALS AND METHODS}

\section{Strains and Culture Conditions}

All strains used in this study are listed in Supplementary Table S1. Anabaena WT and its derivatives were grown in BG11 (Stanier et al., 1971). For heterocyst induction, cells grown to logarithmic phase in BG11 were transferred to BG11 $1_{0}$ (BG11 without combined nitrogen) medium as described previously (Golden et al., 1991). All strains were cultured in liquid medium at $30^{\circ} \mathrm{C}$ with shaking at $180 \mathrm{rpm}$ and the constant illumination with light intensity at $30 \mu \mathrm{molm} \mathrm{m}^{-2} \mathrm{~s}^{-1}$, the $\mathrm{CO}_{2}$ concentration is $400 \mathrm{ppm}$. The overexpression strains based on the artificial CT promoter (Xing et al., 2020) were grown first in BG11 medium to logarithmic phase, then transferred to $B G 11_{0}$ in the presence of copper and theophylline, for the induction of 
heterocysts development and gene expression. When not specified, the concentration of the copper and theophylline is 0.5 and $2 \mathrm{mM}$, respectively. To modify the c-di-GMP level in vivo, gradient concentrations of copper/theophylline were tested at: $0 \mu \mathrm{M} / 0 \mathrm{mM}, 0.125 \mu \mathrm{M} / 0.5 \mathrm{mM}, 0.25 \mu \mathrm{M} / 1 \mathrm{mM}, 0.5 \mu \mathrm{M} / 2 \mathrm{mM}$, and $1 \mu \mathrm{M} / 4 \mathrm{mM}$. Whenever necessary, neomycin $(25 \mu \mathrm{g} / \mathrm{ml})$, streptomycin $(2.5 \mu \mathrm{g} / \mathrm{ml})$ or spectinomycin $(5 \mu \mathrm{g} / \mathrm{ml})$ was supplied in $\mathrm{BG} 11$ or $\mathrm{BG} 11_{0}$ medium.

\section{Construction of Plasmids and Cyanobacterial Recombinant Strain}

All plasmids used in this study are listed in Supplementary Table S2 and verified by Sanger sequencing. All the oligonucleotides used for plasmid construction and mutant checking in this study are listed in Supplementary Table S3.

All mutant stains with markless deletion of the corresponding genes were generated in this study by the genome editing technique based on CRISPR-Cpf1, and the plasmids were constructed as previously described (Niu et al., 2019). The plasmids for gene overexpression in Anabaena were constructed based on the PCT vector (Xing et al., 2020), allowing control of gene expression by the artificial CT promoter. This promoter was consisted of the petE promoter region and a theophylline riboswitch. Gene expression could thus be induced by adding copper and theophylline in the culture media. The plasmids used for protein purification from E. coli BL21 (DE3) were constructed based on the pHTwinStrep vector, which containing two carboxyl-terminals Strep-tag at the C-terminal.

To construct the mutant and overexpression strains, the corresponding plasmid was transferred into Anabaena by conjugation through triparental mating as previously described (ElhaI et al., 1997). All mutants and overexpression strains were confirmed by PCR.

\section{Extraction and Quantification of Cellular c-di-GMP}

c-di-GMP in Anabaena was analyzed as previously described (Agostoni et al., 2013). At the indicated time points or the indicated conditions, $2 \mathrm{ml}$ of cells were quickly collected by filtering under standard light illumination and resuspended with $500 \mu \mathrm{l}$ ice-cold extraction buffer containing 40\% acetonitrile, $40 \%$ methanol, and $0.1 \mathrm{~N}$ formic acid. The cells were broken by crusher FastPrep-24TM5G and the cell lysis efficiency was checked under a microscopy. Once the cells were totally broken, the samples were incubated at $-20^{\circ} \mathrm{C}$ for $30 \mathrm{~min}$ and then centrifuged at $12,000 \mathrm{~g}$ for $5 \mathrm{~min}$ at $4^{\circ} \mathrm{C}$. The supernatant was transferred to a new $1.5 \mathrm{ml}$ tube and stored at $-80^{\circ} \mathrm{C}$ until further analysis. The protein concentrations of all samples were measured in parallel by Bradford assay. All experiments were repeated three times.

For quantification, the supernatants were evaporated and dried by vacuum manifold. The pellets were resuspended in an equal volume of Milli-Q-purified water. $20 \mu \mathrm{l}$ of each sample was analyzed by UPLC-MS/MS on ACQUITY UPLC H-classXevo TQ MS system, equipped with a Waters BEH C18 $2.1 \times 50 \mathrm{~mm}$ column. All samples were filtered with a $0.22 \mu \mathrm{m}$ Ultra free-MC membrane before subjected to the column.
The sample was separated at $25^{\circ} \mathrm{C}$ with a flow rate of $0.3 \mathrm{ml} /$ min and the chromatograph process used was as the following: gradient of solvent A (10 mM tributylamine plus $15 \mathrm{mM}$ acetic acid in 97:3 water: methanol) to solvent $B$ (methanol): $\mathrm{t}=0 \mathrm{~min}$; A-99\%: B- $1 \%, t=2.5 \mathrm{~min} ; \mathrm{A}-80 \%$ : $\mathrm{B}-20 \%, \mathrm{t}=7.0 \mathrm{~min} ; \mathrm{A}-35 \%$ : $\mathrm{B}-65 \%, t=7.5 \mathrm{~min} ; \mathrm{A}-5 \%: \mathrm{B}-95 \%, \mathrm{t}=9.01 \mathrm{~min} ; \mathrm{A}-99 \%: \mathrm{B}-1 \%$, $\mathrm{t}=10 \mathrm{~min}$ (end of gradient). The $\mathrm{c}$-di-GMP was detected with electrospray ionization using multiple reaction monitoring in negative-ion mode at $\mathrm{m} / \mathrm{z} 689.16 \rightarrow 344.31$. The parameters for mass spectrum were as the following: capillary voltage, $3.5 \mathrm{kV}$; cone voltage, $50 \mathrm{~V}$; collision energy, $34 \mathrm{~V}$; source temperature, $110^{\circ} \mathrm{C}$; desolvation temperature, $350^{\circ} \mathrm{C}$; cone gas flow (nitrogen), 50l/h; desolvation gas flow (nitrogen), 800 $/ \mathrm{h}$; collision gas flow (nitrogen), $0.15 \mathrm{ml} / \mathrm{min}$; and multiplier voltage, $650 \mathrm{~V}$. Standard c-di-GMP (Biolog) at the concentrations of $500 \mathrm{nM}$, $250 \mathrm{nM}, 125 \mathrm{nM}, 62.5 \mathrm{nM}, 31.25 \mathrm{nM}, 15.62 \mathrm{nM}$, and $7.81 \mathrm{nM}$ were used to generate the standard curve for calculating the c-di-GMP concentration of each sample.

\section{Microscopy}

The microscope images were captured by SDPTOP EX30 microscope. Microscope images taken at the indicated times and conditions were used for analyzing the heterocyst frequency and vegetative cell intervals. All experiments were analyzed with three parallel analyses.

\section{Quantitative Real-Time PCR}

Wide-type Anabaena was cultivated to the log phase in BG11 and then transferred to BG1 $1_{0}$ medium for heterocyst induction as described previously (Golden et al., 1991). To prepare the samples, $30 \mathrm{ml}$ of Anabaena were harvested at $0,3,16,48$, and $72 \mathrm{~h}$ after nitrogen starvation, respectively, in triplicate. The total RNA was extracted with Plant Total RNA Isolation Kit (Foregene), and the genomic DNA was removed by treating with RNase-free DNase I (Promega, United States). The obtained total RNA samples were incubated with reverse transcriptase with HiScript Q RT super mix (Vazyme).

Quantitative Real-Time PCR was performed by using ChamQ SYBR qPCR Master Mix (Vazyme) with specific primers listed in Supplementary Table S3. The house keeping gene $\operatorname{rnpB}$ was used as an internal control. Primers Pallrs04F334 and Pallrs04R456 (for $r n p B$ ), PcdgS4F226 and PcdgSR402 (for $c d g S$ ), and PcdgSHF2342 and PcdgSHR2455 (for $c d g S H$ ), were used to amplify specific fragments. The RT-PCR products were confirmed by sequencing. Data were analyzed by ABI 7500 SDS software. To be consistent, the baseline was set automatically by the software. The comparative CT method ( $2^{-\triangle \triangle \mathrm{CT}}$ method) was used to analyze the expression of $c d g S$ and $c d g S H$. The data correspond to relative mRNA expression levels, shown as the mean $\pm \mathrm{SE}$ (standard error) of three replicates $(N=3)$ analyzed by $t$ test, and the significance was $p<0.05$.

\section{Protein Expression and Purification}

The plasmids carrying recombinant genes for Strep-tag fusion proteins were transformed into competent cells of $E$. coli BL21 (DE3; Novagen) and then grew in LB medium with kanamycin 
$(50 \mu \mathrm{g} / \mathrm{ml})$. When the cell culture reached $\mathrm{OD}_{600}=0.5-0.6$, $0.5 \mathrm{mM}$ IPTG was added and continued to grow overnight at $18^{\circ} \mathrm{C}$. Cells were harvested by centrifugation at $8000 \mathrm{~g}$ for $5 \mathrm{~min}$, resuspended in buffer $\mathrm{W}(100 \mathrm{mM}$ Tris- $\mathrm{HCl} \mathrm{pH} 8.0,1 \mathrm{M} \mathrm{NaCl}$ and $1 \mathrm{mM}$ EDTA) and lysed by French press. The lysate was cleared by centrifugation at $12000 \mathrm{~g}$ for $30 \mathrm{~min}$ at $4^{\circ} \mathrm{C}$, and the supernatant was incubated with buffer $\mathrm{W}$ equilibrated Strep-Tactin $^{\circledR}$ XT Superflow ${ }^{\circledR}$ resin (IBA) at $4^{\circ} \mathrm{C}$ for $1 \mathrm{~h}$. The resin was then collected by filtration and washed with 10 column volumes $(\mathrm{CV})$ of buffer $\mathrm{W}$. The proteins were eluted with elution buffer (buffer $\mathrm{W}$ containing $50 \mathrm{mM}$ biotin) and dialyzed against $50 \mathrm{mM}$ Tris- $\mathrm{HCl}(\mathrm{pH} 8.0), 10 \mathrm{mM} \mathrm{MgCl}$, $0.5 \mathrm{mM}$ EDTA, and $300 \mathrm{mM} \mathrm{NaCl}$ for $12 \mathrm{~h}$ at $4^{\circ} \mathrm{C}$.

\section{In vitro Assays of DGC and PDE Activity}

The DGC and PDE activity assay was analyzed as previously described (Enomoto et al., 2015). In brief, the total volume of each reaction was $50 \mu \mathrm{l}$ and the reaction mixture contained $50 \mathrm{mM}$ Tris. $\mathrm{HCl} \mathrm{pH} 8.0,10 \mathrm{mM} \mathrm{MgCl}_{2}, 0.5 \mathrm{mM}$ EDTA, $300 \mathrm{mM}$ $\mathrm{NaCl}, 50 \mu \mathrm{M}$ GTP (Thermo scientific) for DGC or $50 \mu \mathrm{M}$ c-di-GMP (Biolog) for PDE. Each reaction was initiated by adding the corresponding protein at a final concentration $2 \mu \mathrm{M}$, and then incubated at $25^{\circ} \mathrm{C}$ for $30 \mathrm{~min}$. All the reactions were stopped by adding $20 \mathrm{mM}$ EDTA and immediately heated at $95^{\circ} \mathrm{C}$ for $5 \mathrm{~min}$. Before subjected to HPLC analysis, all samples were filtered with a $0.22 \mu \mathrm{m}$ Ultrafree-MC membrane by centrifugation. Nucleotides were separated by reverse-phase HPLC through a C18 column $(150 \mathrm{~mm} \times 6 \mathrm{~mm}$ i.d.; DAISOPAK SP-120-5- ODS-AP; Daiso). $20 \mu \mathrm{l}$ sample from each reaction was injected and eluted with buffer A $(50 \mathrm{mM}$ potassium phosphate, $4 \mathrm{mM}$ tetrabutylammonium hydrogen sulfate, $\mathrm{pH}$ 6.0) and buffer B (75\% buffer A, 25\% methanol, V/V) at $1.2 \mathrm{ml} \mathrm{min}^{-1}$, and the elution protocol as followed: $0 \mathrm{~min}, 80 \% / 20 \%$ $\mathrm{A} / \mathrm{B} ; 2.5 \mathrm{~min}, 80 \% / 20 \% \mathrm{~A} / \mathrm{B} ; 5 \mathrm{~min}, 70 \% / 30 \% \mathrm{~A} / \mathrm{B} ; 14 \mathrm{~min}$, $60 \% / 40 \% \mathrm{~A} / \mathrm{B} ; 25 \mathrm{~min}, 0 \% / 100 \% \mathrm{~A} / \mathrm{B} ; 32 \mathrm{~min}, 0 \% / 100 \% \mathrm{~A} / \mathrm{B}$; $33 \mathrm{~min}, 80 \% / 20 \% \mathrm{~A} / \mathrm{B}$; and $34 \mathrm{~min}, 80 \% / 20 \% \mathrm{~A} / \mathrm{B}$. The nucleotides were detected at $254 \mathrm{~nm}$. Standard c-di-GMP and GTP at concentrations of $10,20,40,80,160,320$, and $640 \mu \mathrm{M}$ in the reaction buffer was used to generate the standard curve for quantifying the c-di-GMP and GTP levels, respectively, in reactions.

\section{RESULTS}

\section{c-di-GMP Level Increases Transiently During Heterocysts Development}

To investigate the relationship between c-di-GMP and heterocysts development, we first determined the changes of intracellular concentration of c-di-GMP during this physiological process in Anabaena. c-di-GMP from wild-type (WT) cultures at different time points after nitrogen starvation was extracted and quantified by ultra-high performance liquid chromatography with tandem mass spectrometry detection (UPLC-MS/MS). As shown in Figure 1, the c-di-GMP level in cells in BG11 medium (time 0) was $0.078 \pm 0.014 \mathrm{nmol} / \mathrm{mg}$ protein. After exposure of cells to BG11 $1_{0}$ medium, c-di-GMP levels increased by 3-fold at $3 \mathrm{~h}$ after the transfer, reaching a peak level of $0.231 \pm 0.034 \mathrm{nmol} /$ mg protein. After this time point, the levels of c-di-GMP gradually decreased to the basal level in $24 \mathrm{~h}$. These results suggested that c-di-GMP may be an important signaling molecule during heterocyst differentiation.

\section{$\Delta c d g S H$ Mutant Caused an Increase in Heterocyst Frequency}

To investigate the role of c-di-GMP in heterocyst differentiation, in-frame markerless deletion of all 16 genes encoding proteins with predicted DGC and/or PDE domains were generated by genome editing technique based on CRISPR-Cpf1 (Supplementary Figure S1; Niu et al., 2019). We determined the ability of heterocyst formation for all mutants grown in BG1 $1_{0}$. When observed under the microscope, 2 of the 16 mutants, $\Delta c d g S H$ and $\Delta c d g S$, were affected in heterocyst frequency while all others had a heterocyst pattern and percentage comparable to those of the WT (Table 1). The phenotype of $\Delta c d g S$ was similar to that already reported (Neunuebel and Golden, 2008). Indeed, heterocyst frequency of the mutant was similar to that of WT at $24 \mathrm{~h}$ after the induction $(8.0 \pm 0.27 \%)$ and dropped by $60 \%(2.5 \pm 0.08 \%) 3$ days later after the induction as compared to the WT $(6.2 \pm 0.16 \%)$. It can grow in BG1 $1_{0}$, but less well than the WT (Supplementary Figure S6B). While $c d g S$ was required to maintain heterocyst frequency over time, without affecting the initial heterocyst pattern $\Delta c d g S H$ showed a very different phenotype. After $24 \mathrm{~h}$ of growth in $\mathrm{BG} 11_{0}$

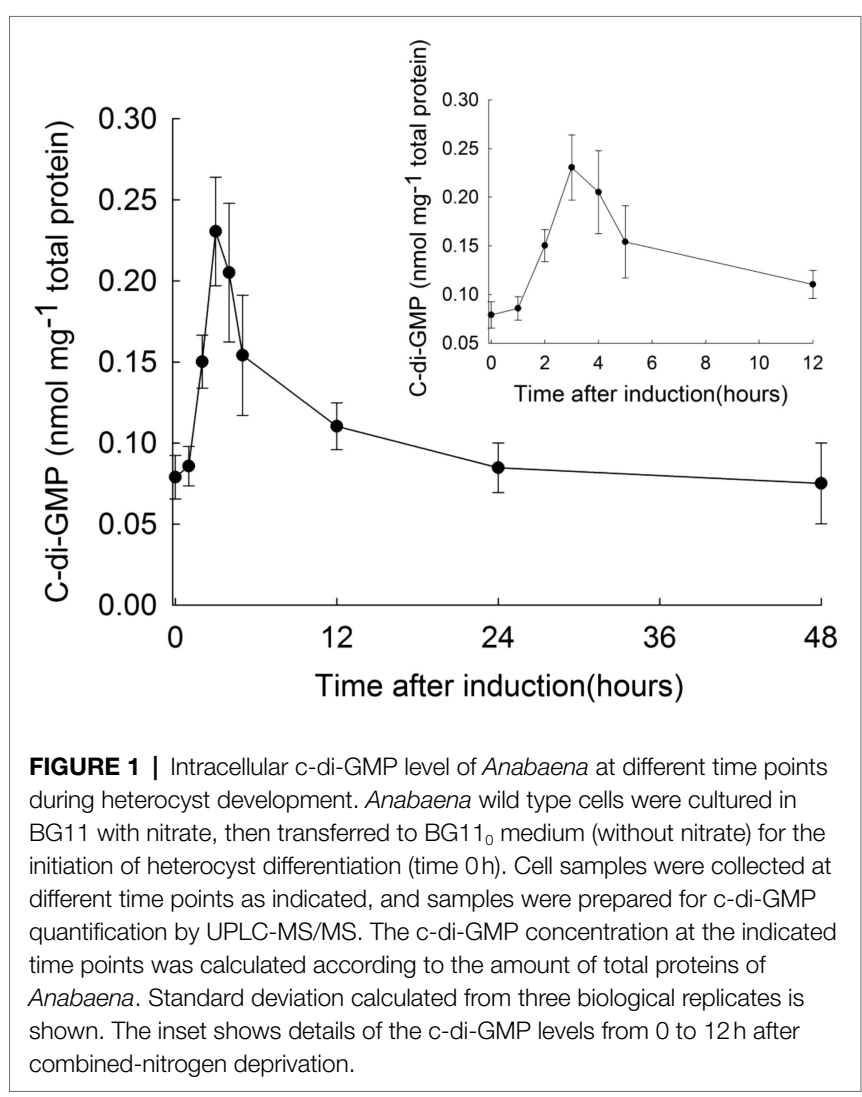


TABLE 1 | Heterocyst frequency of 16 mutants affecting c-di-GMP synthesis or hydrolysis at 24 and $72 \mathrm{~h}$ after nitrogen starvation.

\begin{tabular}{|c|c|c|}
\hline Strain & $\begin{array}{l}\text { Heterocyst frequency } \\
24 \mathrm{~h}\end{array}$ & $\begin{array}{c}\text { Heterocyst frequency } \\
72 \mathrm{~h}\end{array}$ \\
\hline$W T$ & $8.0 \pm 0.33 \%$ & $6.2 \pm 0.16 \%$ \\
\hline$\Delta$ all1012 & $7.8 \pm 0.12 \%$ & $5.8 \pm 0.41 \%$ \\
\hline$\Delta$ all1219 & $7.9 \pm 0.30 \%$ & $6.1 \pm 0.31 \%$ \\
\hline$\Delta$ all2416 & $7.8 \pm 0.24 \%$ & $5.9 \pm 0.43 \%$ \\
\hline$\Delta$ all2874 (cdgS) & $8.0 \pm 0.27 \%$ & $2.5 \pm 0.08 \%$ \\
\hline$\Delta$ alr3504 & $8.1 \pm 0.46 \%$ & $5.6 \pm 0.56 \%$ \\
\hline$\Delta$ alr3599 & $8.3 \pm 0.16 \%$ & $6.1 \pm 0.32 \%$ \\
\hline$\Delta$ all4896 & $8.1 \pm 0.41 \%$ & $5.7 \pm 0.18 \%$ \\
\hline$\Delta$ all5174 & $7.7 \pm 0.19 \%$ & $6.1 \pm 0.42 \%$ \\
\hline$\Delta$ alr1230 & $8.2 \pm 0.54 \%$ & $6.2 \pm 0.18 \%$ \\
\hline$\Delta$ all0219 & $8.0 \pm 0.37 \%$ & $6.1 \pm 0.38 \%$ \\
\hline$\Delta$ all1175 (cdgSH) & $10.5 \pm 0.43 \%$ & $6.0 \pm 0.27 \%$ \\
\hline$\Delta$ all4225 & $7.6 \pm 0.22 \%$ & $6.2 \pm 0.33 \%$ \\
\hline$\Delta$ all4897 & $8.2 \pm 0.11 \%$ & $5.6 \pm 0.24 \%$ \\
\hline$\Delta$ alr2306 & $8.1 \pm 0.29 \%$ & $5.8 \pm 0.28 \%$ \\
\hline$\Delta$ alr3170 & $8.1 \pm 0.42 \%$ & $6.0 \pm 0.32 \%$ \\
\hline$\Delta$ alr3920 & $8.3 \pm 0.03 \%$ & $6.2 \pm 0.21 \%$ \\
\hline
\end{tabular}

medium, $\Delta c d g S H$ mutant could differentiate mature heterocysts; however, the percentage of heterocysts showed an increase $(10.3 \% \pm 0.43 \%)$ when compared to WT $(8.0 \% \pm 0.33 \%)$, and a Mch (multiple-contiguous-heterocysts) phenotype was occasionally observed (Figures 2A,B). To confirm this phenotype, we further quantified the pattern of heterocysts along the filaments. Heterocyst intervals of WT show regularity, with 11 to 13 vegetative cells as the most representative intervals. In $\Delta c d g S H$ mutant, the distribution still kept a regularity, but the intervals shortened to 8 to 10 vegetative cells as the most representative ones (Figure 2B). Interestingly, from $48 \mathrm{~h}$ after induction, the heterocyst frequency of the $\Delta c d g S H$ mutant became similar to WT (Figure 2C), indicating that the mutation affected only the initial establishment of the heterocyst frequency, but not its maintenance. The $\Delta c d g S H$ can grow well in BG1 $1_{0}$ (Supplementary Figure S6A). The phenotype of $\Delta c d g S H$ could be complemented by $c d g S H$ controlled by its own promoter present on a replicative plasmid (Figure 2C, strain $\mathrm{C} c d g S H$ ). Taken together, among the 16 annotated genes related to c-di-GMP levels on the genome, $c d g S H$ and $c d g S$ appear to be the major ones involved in the regulation of heterocyst pattern.

\section{CdgSH and CdgS Have Additive Effect for Regulating Heterocyst Frequency}

Although both $c d g S H$ and $c d g S$ regulate heterocyst intervals, their phenotypes were very distinct. To investigate the relationship between $c d g S H$ and $c d g S$ in regulating heterocyst intervals, we constructed a $\Delta c d g S H \Delta c d g S$ double mutant. $24 \mathrm{~h}$ following combined-nitrogen deprivation, heterocyst frequency of this double mutant was $10.5 \% \pm 0.21 \%$, similar as $\Delta c d g S H$. However, with prolonged time in combined-nitrogen free medium, the frequency decreased gradually, and reaching $5.2 \% \pm 0.19,2.5 \% \pm 0.17$ and $2.1 \% \pm 0.14 \%$ at 48,72 , and $96 \mathrm{~h}$, respectively (Figure 2C), a phenotype similar as $\Delta c d g S$. Although the two single mutants displayed opposite effect on the frequency of heterocysts, the results indicated that the phenotype of the double mutant was the sum of the single ones under diazotrophic condition. This indicated that these two genes acted independently at different time points during heterocyst development, with $c d g S H$ for establishment of heterocyst pattern (the first 24h), while $c d g S$ for maintenance of the frequency of heterocysts at the later stages.

Next, to better understand how the two genes could participate at different time points in heterocyst development, we checked the transcription levels of $c d g S H$ and $c d g S$ in WT at different time points after nitrogen starvation by quantitative Real-Time PCR. Our data indicated that these two genes showed opposite transcriptional profiles (Figure 2D). Following nitrogen stepdown, the transcript level of $c d g S$ was kept steady in the first $3 \mathrm{~h}$, and then increased after $16 \mathrm{~h}$ post induction. At $72 \mathrm{~h}$ after combined-nitrogen deprivation, the relative transcript level of $c d g S$ was increased around 3 -fold. In contrast, under the same conditions, the transcriptional activity of $c d g S H$ was dropped to nearly undetectable level at $16 \mathrm{~h}$ after transfer to $\mathrm{BG} 11_{0}$. Although gradually increased after $16 \mathrm{~h}$, it still remained at a much lower at $72 \mathrm{~h}$ as compared to that observed at time 0 (Figure 2D). The transcriptional profiles of these two genes are in good agreement with the phenotype of their corresponding mutants. Indeed, $c d g S H$ had a higher transcriptional activity at the onset of heterocyst induction, and its inactivation affected the establishment of heterocyst frequency. In contrast, the transcriptional activity of $c d g S$ was low at the beginning of heterocyst induction, but increased steadily afterwards; consistently, heterocyst frequency continuously dropped from the second day overtime after combined-nitrogen deprivation.

\section{CdgSH Is a Bi-Functional Enzyme With a Dominating PDE Activity in vitro}

$\mathrm{CdgSH}$ is predicted to be a multi-domain cytoplasmic protein, with FHA, PAS, GAF, GGDEF and EAL domains arranged in tandem (Supplementary Figure S1). The GGDEF and the EAL domains are both located towards the C-terminus of the polypeptide and contain all the conserved residues required for their corresponding catalytic activity (Supplementary Figure S2). This observation suggests that $\mathrm{CdgSH}$ may exhibit both c-di-GMP synthesis and degradation activities. To check this observation, $\mathrm{CdgSH}$ and its variants were expressed in E. coli BL21 (DE3) and purified using a strep-tag affinity column (Supplementary Figure S3). The DGC and PDE activities of these proteins were then determined. Our results showed that no matter which of the substrates, GTP or c-di-GMP, was used, only one product, pGpG, could be detected by HPLC (Figure 3). This indicated that $\mathrm{CdgSH}$ could synthesize c-di-GMP from GTP, and immediately hydrolyzed c-di-GMP to pGpG. To further confirm this observation, the $\mathrm{CdgSH}^{\mathrm{GGAAF}}$ variant, which the catalytic and the metal ion coordination sites were replaced (mutations D530A and E531A), was produced (Chan et al., 2004; Wassmann et al., 2007). This variant kept an intact PDE domain, but a defective DGC domain. As shown in Figure 3, $\mathrm{CdgSH}^{\mathrm{GGAAF}}$ could only degrade c-di-GMP to pGpG, and when GTP was added, no activity was detected. The $\mathrm{CdgSH}^{\mathrm{AAA}}$ variant, which inactivated the PDE activity of $\mathrm{CdgSH}$ by mutating the metal ion coordination sites (mutations E656A and L658A; 


\section{A}

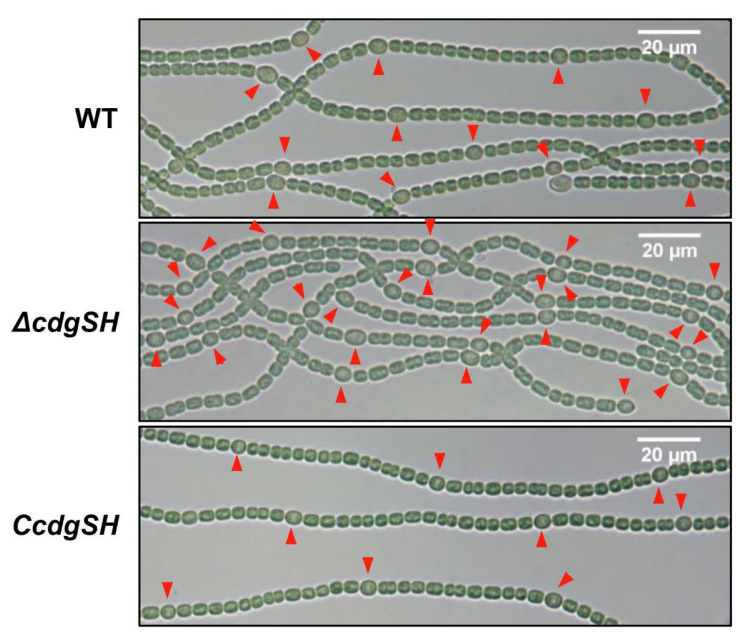

B

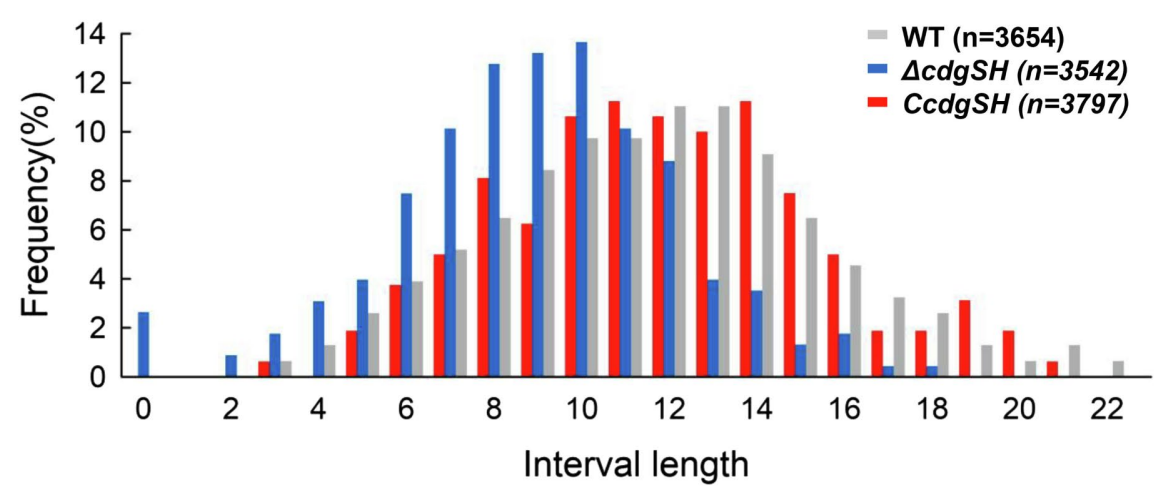

C

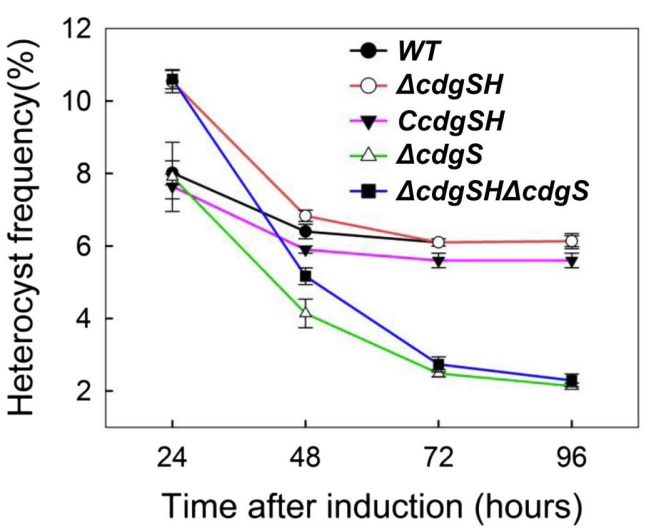

D

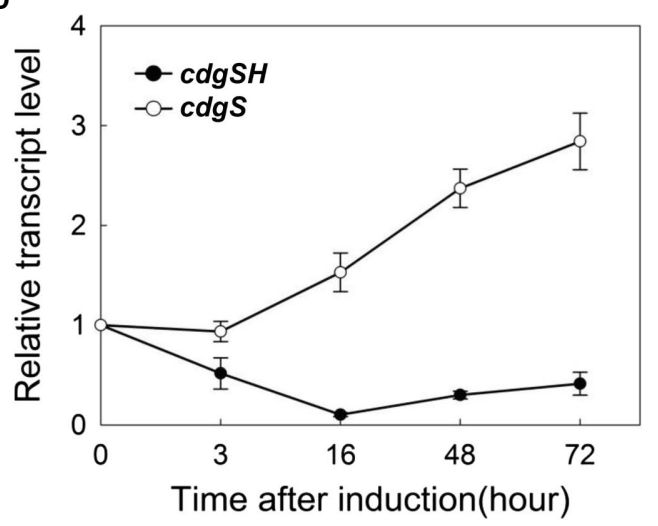

FIGURE 2 | Deletion of 2 genes (cdgSH and cdgS) involved in c-di-GMP synthesis or hydrolysis affects heterocyst frequency. (A) Light microscopic images of filaments from wild type (WT), $c d g S H$-deletion strain $(\Delta c d g S H)$, and the complemented strain (CcdgSH, corresponding to $\Delta c d g S H$ carrying the cdgSH gene on a replicative plasmid), at $24 \mathrm{~h}$ after the initiation of heterocyst differentiation induced by deprivation of combined nitrogen. Heterocysts are indicated by red triangle. (B) Analysis of heterocyst pattern in different strains as in (A) at $24 \mathrm{~h}$ after initiation of heterocyst differentiation. The number of vegetative cells between two heterocysts (interval length) was counted, and the distribution of heterocyst intervals was analyzed according to the frequency of each interval. The number of the quantified vegetative cells is indicated in parentheses above the graph. (C) The heterocyst frequency of the indicated strains at different time points after nitrogen stepdown (24, 48, 72, and 96h). (D) The transcription levels of cdgSH and cdgS in WT. Samples were taken at the indicated time point after nitrogen stepdown, and total RNAs were extracted, and analyzed by quantitative PCR (qPCR). All the values are showed as mean \pm standard deviation, calculated from three biological replicates.

Barends et al., 2009; Tchigvintsev et al., 2010), was also tested, and it could synthesize c-di-GMP from GTP, but the c-di-GMP hydrolysis activity was abolished (Figure 3).
To further investigate the relationship between the PDE and DGC activities of $\mathrm{CdgSH}$, we quantified the steady-state kinetic parameters ( $k c a t$ and $\mathrm{Km}$ ) for $\mathrm{CdgSH}, \mathrm{CdgSH}^{\mathrm{GAAEF}}$ and 
A

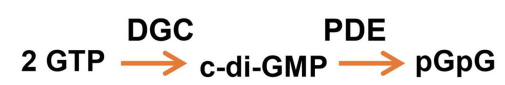

B

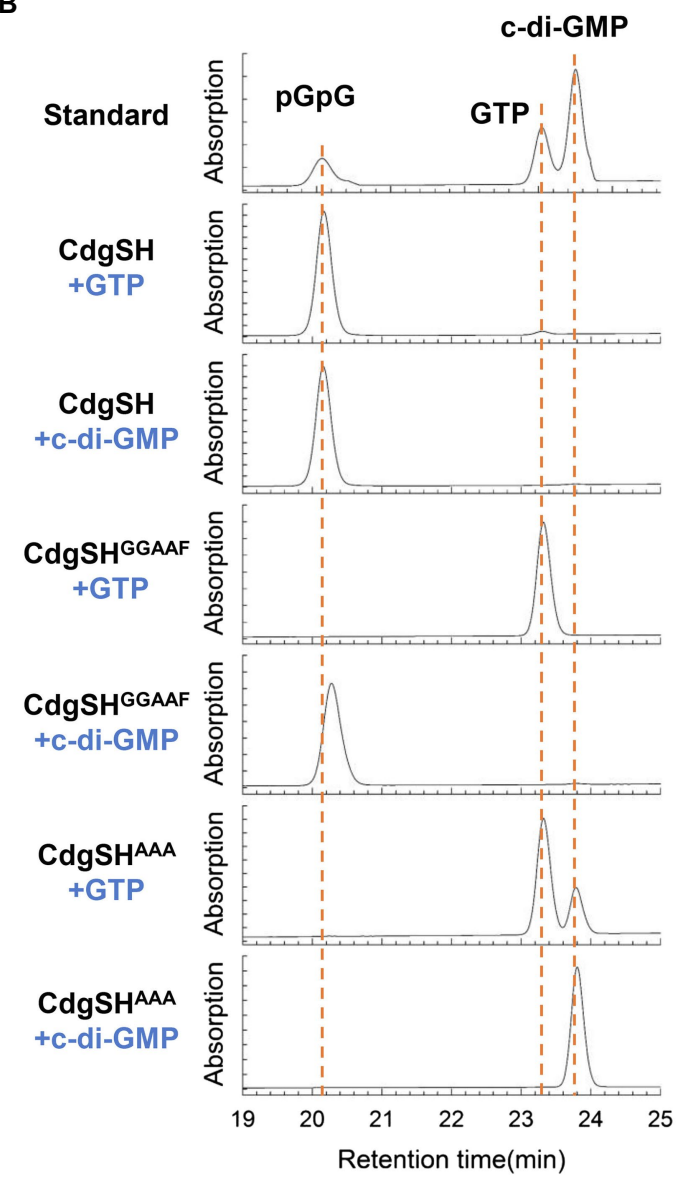

FIGURE 3 | Anabaena protein CdgSH has both diguanylate cyclase (DGC) and c-di-GMP-specific phosphodiesterase (PDE) activities. (A) Scheme showing the reactions catalyzed by the PDE (c-di-GMP $\rightarrow$ pGpG) and DGC (2 GTP $\rightarrow$ C-di-GMP) activities of an enzyme. (B) The enzymatic activities of

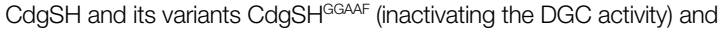
$\mathrm{CdgSH}^{\text {AAA }}$ (abolishing the PDE activity) were evaluated by the reaction products. Recombinant proteins were produced from E. coli, purified, and tested. All reaction substrates or products were assessed by the retention time using HPLC according to the standards. The top panel shows the retention time of the standard nucleotides: pGpG, c-di-GMP, and GTP. The retention time of each nucleotide was first determined by HPLC separately (data not shown here). The proteins used for PDE and DGC activity assay were indicated at the left of the chromatograms, and the substrate for each reaction was indicated under the name of proteins. CdgSH can convert substrates, GTP and c-diGMP, to pGpG. PDE active-site mutant protein $\mathrm{CdgSH}^{\mathrm{AAA}}$ can only convert GTP to c-di-GMP, and the CdgSH ${ }^{\text {GGAAF}}$, which inactivates the DGC activity of CdgSH, can only hydrolyze c-di-GMP to pGpG.

$\mathrm{CdgSH}^{\mathrm{AAA}}$ under the same experimental conditions. As shown in Table 2, the kcat value for the PDE activity is three times that of the DGC activity in CdgSH, while the DGC $\mathrm{Km}$ values is 1.5 times that of the PDE activity. This result indicates that although the binding efficiencies of the two catalytic domains to their respective substrate are similar, the conversion efficiencies are quite different. Indeed, the catalytic activity of PDE in $\mathrm{CdgSH}$, expressed by the $\mathrm{kcat} / \mathrm{Km}$ ratio is $5.4 \times 10^{-2} \mu \mathrm{M} / \mathrm{S}^{-1}$ (Table 2), which is more than 5 -fold higher than that of DGC $\left(1.0 \times 10^{-2} \mu \mathrm{M} / \mathrm{S}^{-1}\right)$. In addition, we found that the catalytic activity $(\mathrm{kcat} / \mathrm{Km})$ of $\mathrm{PDE}$ in $\mathrm{CdgSH}$ and $\mathrm{CdgSH}^{\mathrm{GGAAF}}$ are similar to each other, suggesting that the PDE activity of CdgSH was independent of the DGC activity. On the other hand, the catalytic activity $(\mathrm{kcat} / \mathrm{Km})$ of DGC in $\mathrm{CdgSH}^{\mathrm{AAA}}$ decreased 3-fold when compared to that of $\mathrm{CdgSH}$, suggesting the existence of a feedback inhibition of the DGC activity by c-di-GMP. Such a phenomenon was known for typical GGDEF domains, which can be explained by the presence of the conserved inhibitory site (RXXD motif) present in $\mathrm{CdgSH}$ which allows c-di-GMP binding and allosteric feedback inhibition (Supplementary Figure S2; Chan et al., 2004; Wassmann et al., 2007). Taken all together, our results demonstrated that CdgSH was a bi-functional enzyme with both DGC and PDE activities, but its PDE activity dominated over the DGC activity, making the c-di-GMP hydrolysis a prevailing output at the end.

For comparison, we also tested the enzymatic activity of CdgS which possesses an N-terminal REC domain and a C-terminal GGDEF domain under the same experimental conditions (Supplementary Figures S1, S2). Our results indicated that CdgS could convert the substrate GTP to c-di-GMP (Supplementary Figure S4), consistent with the result already published (Neunuebel and Golden, 2008). When the catalytic site and the metal ion coordination site (E229A, and E230A) of DGC was replaced (Chan et al., 2004; Wassmann et al., 2007), the c-di-GMP synthesis activity of $\mathrm{CdgS}^{\mathrm{GGAAF}}$ was completely abolished (Supplementary Figure S4). Therefore, $\mathrm{CdgS}$ is a typical c-di-GMP synthase.

\section{$\triangle c d g S H$ Mutant Phenotype Can Be Complemented by Expression of a Heterologous PDE-Encoding Gene, but Not a Heterologous DGC-Encoding Gene}

$\mathrm{CdgSH}$ is a protein containing other domains in addition to the two with opposing activities in c-di-GMP synthesis and hydrolysis. To evaluate the contribution of the different activities of $\mathrm{CdgSH}$ in regulating heterocyst frequency, we examined the ability of heterologous genes encoding c-di-GMP metabolic enzymes to complement the $\Delta c d g S H$ mutant. YdeH (DGC) and $\mathrm{YhjH}$ (PDE) are well-studied c-di-GMP metabolic enzymes from E. coli with high catalytic activity in vitro (Pesavento et al., 2008). Each of the two proteins contains only one catalytic domain, without additional regulatory domains (Supplementary Figure S2). Therefore, they are often used to alter the intracellular c-di-GMP level of various bacteria (Tschowri et al., 2014; Li et al., 2018). To examine their activities in Anabaena, plasmids containing $y d e H$ or $y h j H$ under the control of inducible regulatory elements (the CT promoter) was transferred into Anabaena by conjugation, giving the strain $\mathrm{OE}_{\mathrm{CT}^{-}} y d e H$ or $\mathrm{OE}_{\mathrm{CT}^{-}}-y h j H$, respectively. We then determined the intracellular c-di-GMP levels of the two recombinant stains. Our data revealed that the intracellular c-di-GMP level of the 
TABLE 2 | The steady-state kinetics of CdgSH and its variants CdgSH GGAAF (abolishing the synthesis activity) and CdgSH ${ }^{\text {AAA }}$ (abolishing the hydrolysis activity).

\begin{tabular}{|c|c|c|c|c|c|c|}
\hline \multirow{2}{*}{ Enzyme } & \multicolumn{3}{|c|}{ DGC (GTP $\rightarrow$ c-di-GMP) } & \multicolumn{3}{|c|}{ PDE (c-di-GMP $\rightarrow$ pGpG) } \\
\hline & $\mathrm{Km}(\mu \mathrm{M})$ & Kcat $\left(\mathrm{s}^{-1}\right)$ & Kcat/Km $\left(\mu \mathrm{Ms}^{-1}\right)$ & $\mathrm{Km}(\mu \mathrm{M})$ & Kcat $\left(\mathrm{s}^{-1}\right)$ & $\mathrm{Kcat} / \mathrm{Km}\left(\mu \mathrm{Ms}^{-1}\right)$ \\
\hline $\mathrm{CdgSH}$ & 37.2 & 0.39 & $1.05 \times 10^{-2}$ & 23.5 & 1.26 & $5.4 \times 10^{-2}$ \\
\hline $\mathrm{CdgSH}^{\mathrm{GGAAF}}$ & - & - & - & 31.0 & 1.78 & $5.7 \times 10^{-2}$ \\
\hline $\mathrm{CdgSH}^{\mathrm{AAA}}$ & 34.8 & 0.12 & $3.45 \times 10^{-3}$ & - & - & - \\
\hline
\end{tabular}

two recombinant strains, growing in BG11 medium and induced by adding $2 \mathrm{mM}$ theophylline and $0.5 \mu \mathrm{M} \mathrm{Cu} \mathrm{Cu}^{2+}$, displayed significant changes as compared with WT after $24 \mathrm{~h}$ of induction. The c-di-GMP level of the cells in $\mathrm{OE}_{\mathrm{CT}}-y d e H$ was at $0.29 \pm 0.04 \mathrm{nmol} / \mathrm{mg}$ total protein, corresponding to an increase by 2 -fold relative to that of WT. Under similar conditions, cells of $\mathrm{OE}_{\mathrm{CT}}-y h j H$ decreased the level of c-di-GMP by 2 -fold, to $0.06 \pm 0.01 \mathrm{nmol} / \mathrm{mg}$ total protein (Figure 4A). Therefore, $\mathrm{YdeH}$ and $\mathrm{YhjH}$ are active in Anabaena, and their production could modify the intracellular levels of c-di-GMP in this organism.

Next, we analyzed the heterocyst pattern and frequency of the strains expressing $y h j H$ or $y d e H$ in the $\Delta c d g S H$ background. To make the data comparable, we used the native promoter of $c d g S H$ to drive the expression of $y h j H$ or $y d e H$ on a replicative plasmid. We found the heterocyst pattern in the recombinant strain $\Delta c d g S H:: y h j H$ was restored to the WT pattern, with 11 to 13 vegetative cells as the most representative intervals after $24 \mathrm{~h}$ combined nitrogen step-down and the heterocyst frequency at this time point was also restored to the WT level, to about $8.2 \pm 0.35 \%$ (Figure $4 B$ ). In contrast, the strain $\Delta c d g S H:: y h j H^{\mathrm{AAA}}$ bearing the same plasmid but containing the mutant allele of $y h j H\left(y h j H^{\mathrm{AAA}}\right)$ failed to complement the defect in heterocyst distribution and frequency of $\Delta c d g S H$ (Figure 4B). Furthermore, the -strain $\Delta c d g S H:: y d e H$ was unable to restore the change in heterocyst distribution and frequency of the $\Delta c d g S H$ mutant (Figure 4B; Supplementary Figure S3). Altogether, our results indicated that the c-di-GMP hydrolysis activity of $\mathrm{CdgSH}$, and hence the decrease in c-di-GMP level, was the major element that contributes to the regulation of heterocyst frequency of $\Delta c d g S H$ mutant at $24 \mathrm{~h}$ after nitrogen starvation. With prolonged time in combined-nitrogen free medium, $\Delta c d g S H::$ yhjH strain had a slightly lower heterocyst frequency as compared to the WT from 48 to $96 \mathrm{~h}$ after the transfer to $\mathrm{BG} 11_{0}$ medium (Figure 4C). This phenotype is similar to $\Delta c d g S$ mutant which also displayed a drop in heterocyst frequency as compared to the WT at the same time points. This result could be explained by the fact that the gene yhjH in the complemented strain was carried on a replicative plasmid that is known to lead to a higher level expression than that from the chromosome, and also the fact that $\mathrm{YhjH}$ possess a high PDE activity.

We also tested whether $y d e H$ could complement $\Delta c d g S$. Indeed, the $y d e H$ gene under the control of the native promoter of $c d g S$, carried on a replicative plasmid could complement $\Delta c d g S$ successfully (strain $\Delta c d g S:: y d e H$, Figure 4D). $y d e H^{\mathrm{GGAAF}}$ which encodes an inactive form of $\mathrm{YdeH}$ (E208 and E209, Figure 4D) was unable to complement the phenotype of $\Delta c d g S$ mutant when controlled by the same regulatory system (Figure 4D). These studies indicated that the c-di-GMP metabolic activities of $\mathrm{CdgSH}$ and CdgS were the major reason for the phenotype observed following the inactivation the corresponding genes in Anabaena.

\section{Homeostasis of c-di-GMP Is Critical for Heterocyst Differentiation}

The metabolism of c-di-GMP should be highly regulated during heterocyst development, as demonstrated by the changes of the c-di-GMP levels during the developmental process, as well as the functions of the two genes $c d g S H$ and $c d g S$, acting at different phases of heterocyst differentiation. To check whether such regulation is important, we examined the effect caused by varying the intracellular levels of c-di-GMP. For this purpose, $\mathrm{CdgSH}, \mathrm{CdgS}$, and their corresponding mutant variants were expressed in WT cells of Anabaena, respectively. The expression levels of the genes were titrated by a gradient of inducers as shown in Figure $\mathbf{5}$ and heterocyst development was observed at $24 \mathrm{~h}$ after the initiation by transfer to $\mathrm{BG} 11_{0}$. When $c d g S H$ was tested with increasing concentrations of inducers, heterocyst frequency gradually decreased from 8 to $1.3 \%$. A more pronounced results were obtained when $\mathrm{CdgSH}^{\mathrm{GGAAF}}$ in which the activity of DGC was abolished, since at the highest concentrations of inducers tested, few heterocysts were formed (0.5\%; Figure 5A). These results were consistent with the fact that $\mathrm{CdgSH}$ has both c-di-GMP synthesis and hydrolysis activities, but the hydrolysis activity was dominant, while the $\mathrm{CdgSH}^{\mathrm{GGAAF}}$ variant had only the PDE activity left (Figure 3; Table 2).

Interestingly, adding increasing gradients of inducers to express either the $\mathrm{CdgSH}^{\mathrm{AAA}}$ variant that has only retained the activity of c-di-GMP synthesis or CdgS also led to a reduction of heterocyst frequency. At $24 \mathrm{~h}$ after combined-nitrogen deprivation, with $4 \mathrm{mM}$ theophylline and $1 \mu \mathrm{M} \mathrm{Cu}^{2+}$, the heterocyst frequency of both strains was around $1 \%$, while heterocyst frequency is $8 \%$ without inducers (Figure 5B). Therefore, ectopic expression of genes encoding either c-di-GMP synthase or hydrolase activities repressed heterocyst differentiation.

To further confirm it is the c-di-GMP metabolic activities of $\mathrm{CdgSH}$ that was responsible for such phenotypes, we tested the strain of $\mathrm{OE}_{\mathrm{CT}^{-}} y d e H$ or $\mathrm{OE}_{\mathrm{CT}^{-}} y h j H$ at the same condition, respectively. With no inducers or with low concentrations of $0.5 \mathrm{mM}$ theophylline and $0.125 \mu \mathrm{M} \mathrm{Cu}^{2+}$, the heterocysts of $\mathrm{YdeH}$ and $\mathrm{YhjH}$ overexpression strains were formed with a frequency similar as the WT (Figures 5A,B). However, when the concentrations of inducers increased to $1 \mathrm{mM}$ for theophylline and $0.25 \mu \mathrm{M}$ for $\mathrm{Cu}^{2+}$, the heterocysts frequency of both strains were reduced to about $1 \%$ at $24 \mathrm{~h}$ after the initiation. Heterocyst developments of both strains were completely suppressed when the concentration of the inducers reached for $2 \mathrm{mM}$ theophylline 


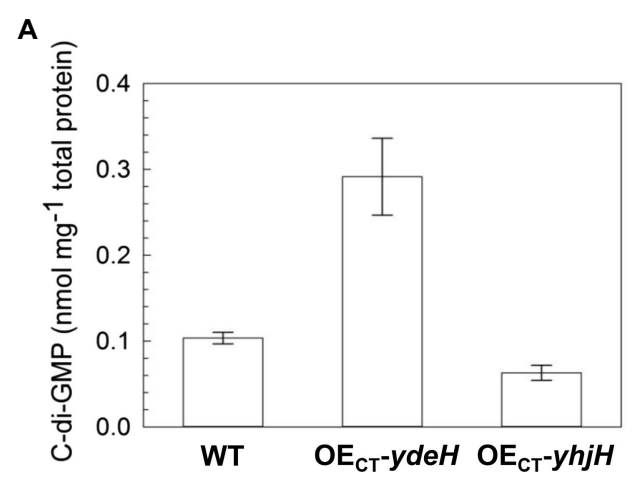

C

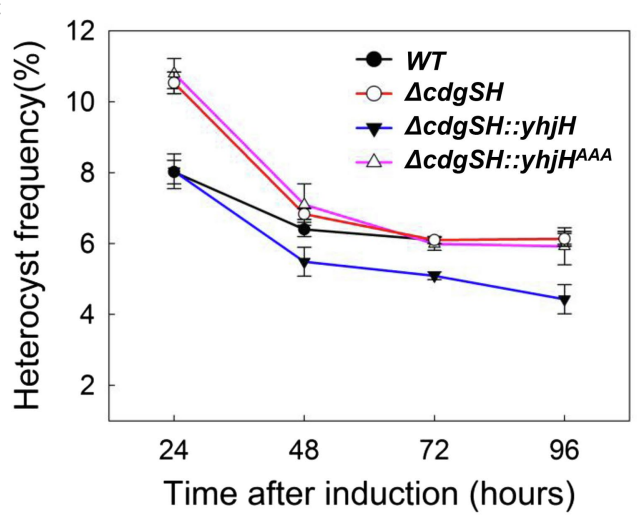

D

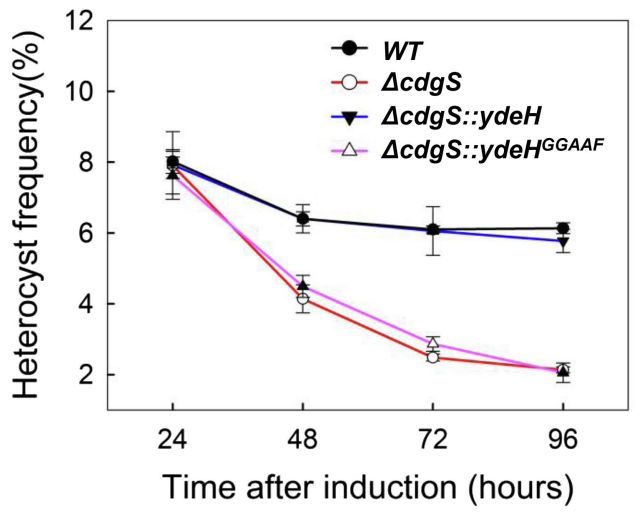

B
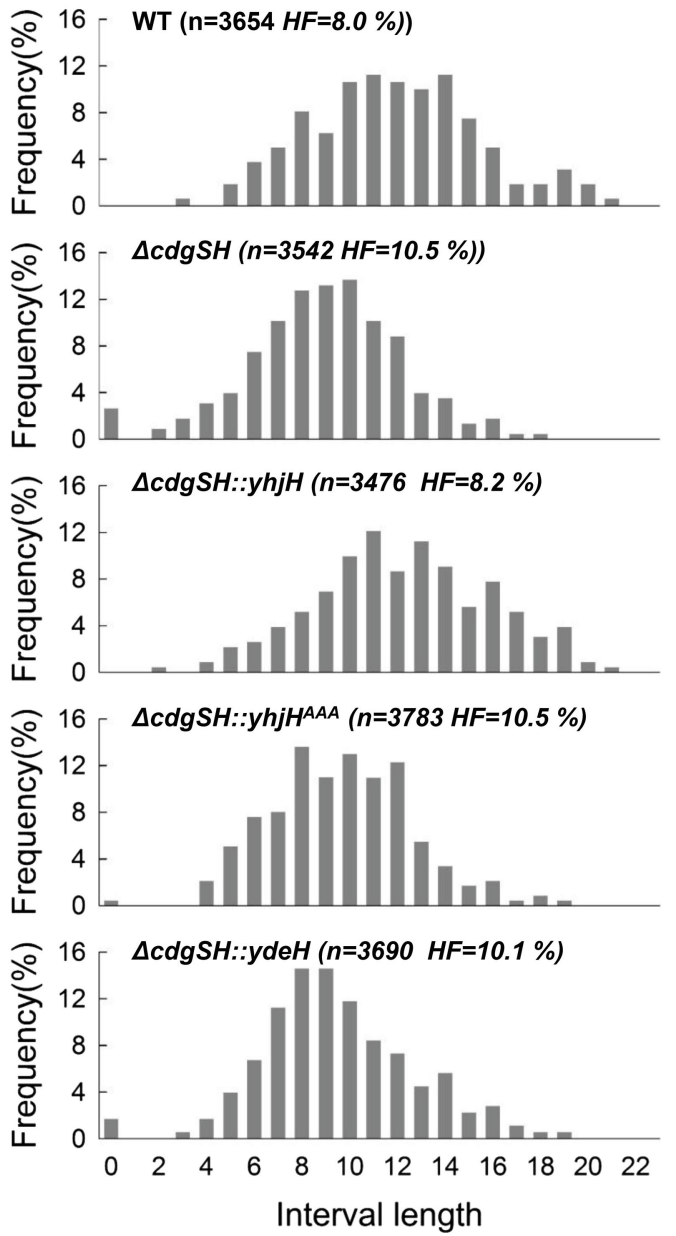

FIGURE 4 | The phenotype of $\Delta c d g S H$ and $\Delta c d g S$ can be complemented by expression of a heterologous PDE (yhjH) and a heterologous DGC (ydeH), respectively. (A) The intracellular c-di-GMP level of the indicated strains, measured at $24 \mathrm{~h}$ after the addition of the inducers $(0.5 \mu \mathrm{M}$ copper and $2 \mathrm{mM}$ theophylline). $\mathrm{OE}_{\mathrm{CT}}-y d e H$, a strain derived from the WT expressing $y d e H$ from $E$. coli under the control of the CT promoter inducible by copper and theophylline; OE $\mathrm{CT}_{-} \mathrm{yhjH}_{\mathrm{H}}$, a WT strain expressing yhjH from E. coli under the control of the CT promoter. All the values of c-di-GMP concentration are expressed as mean \pm standard deviation, calculated from three replicates. (B) Distribution of heterocysts along the filaments analyzed as heterocyst intervals at $24 \mathrm{~h}$ after nitrogen starvation. Interval length represents number of vegetative cells between two heterocysts. Heterocyst frequency (HF) and number of vegetative cells counted at this time point was indicated in parentheses. $\Delta c d g S H:: y h j H$, the $\Delta c d g S H$ mutant complemented by yhjH, a gene from $E$. coli encoding a PDE, under the control of the cdgSH promoter; $\Delta c d g S H:: y h j H A A A$ correspond to $\Delta c d g S H$ carrying $y h j H$ in which the active site EAL was mutated to AAA; $\Delta c d g S H:: y d e H$ is $\Delta c d g S H$ with the expression of $y d e H$ (encoding a DGC enzyme) from E. coli under the control of the cdgSH promoter. (C,D) The heterocyst frequency of the indicated strains at different time points after nitrogen stepdown (24h, 48h, $72 \mathrm{~h}$, and $96 \mathrm{~h})$. All the values of heterocyst frequency are showed as mean \pm standard deviation, calculated from three replicates. In (D), the strain $\triangle$ cdgSH::ydeHGGAAF is similar to $\Delta c d g S H:: y d e H$ except the active site of the DGC activity, GGDEF was mutated to GGAAF.

and $0.5 \mu \mathrm{M} \mathrm{Cu}{ }^{2+}$ (Figures $5 \mathbf{A}, \mathbf{B}$ ). These results were consistent with those obtained by the ectopic expression of the $\mathrm{CdgSH}$, $\mathrm{CdgSH}$ variants and CdgS. To exclude the influence of high dose of inducers or proteins in vivo, effects of production of $\mathrm{CdgSH}^{\mathrm{GGAAF}-\mathrm{AAA}}, \mathrm{CdgS}^{\mathrm{GGAAF}} \mathrm{YdeH}^{\mathrm{GGAAF}}$, and $\mathrm{YhjH}^{\mathrm{AAA}}$ variants, which c-di-GMP synthesis or degradation activities were abolished, as well as WT strain were also examined under the same experimental conditions (Figure 5C; Supplementary Figure S5A). Even at the highest concentrations of the two inducers added, heterocyst frequency and pattern were hardly affected. 
A

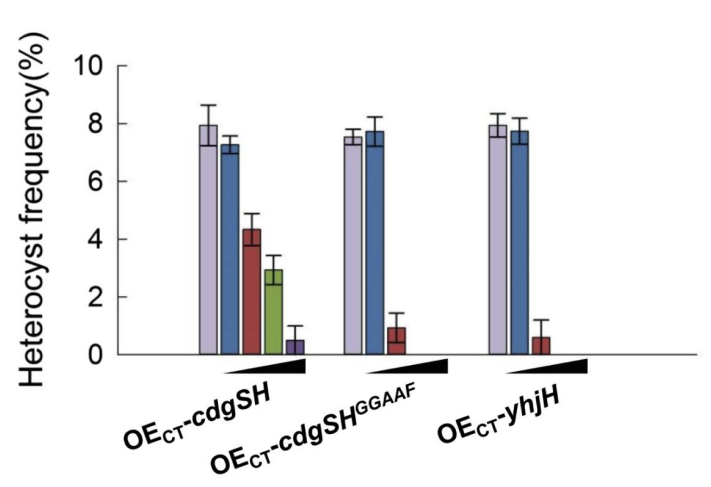

B

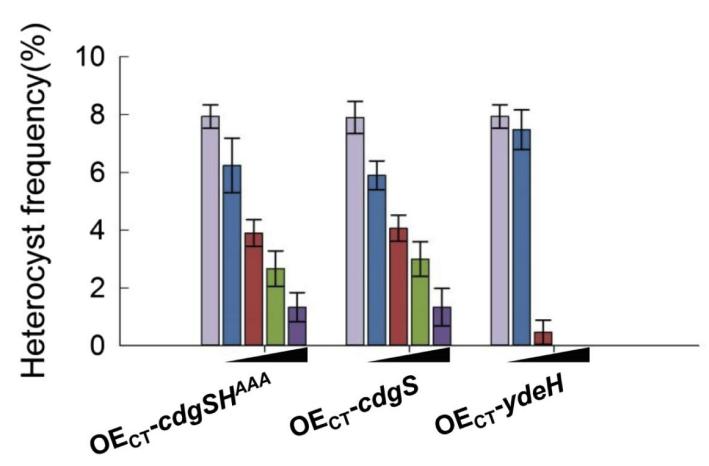

C

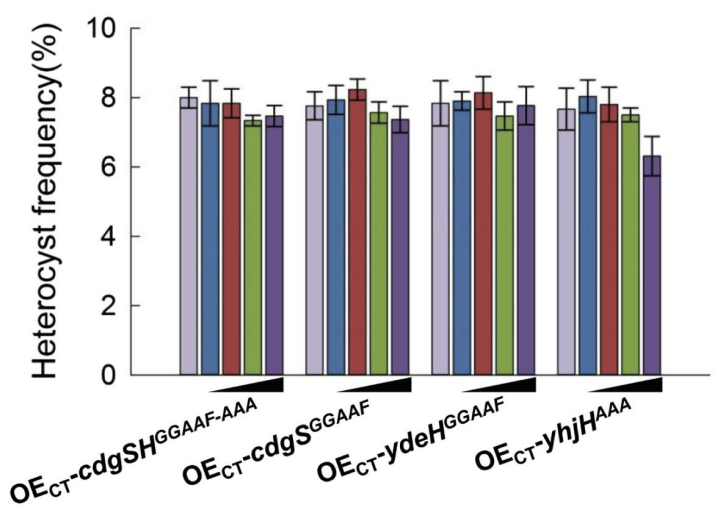

D

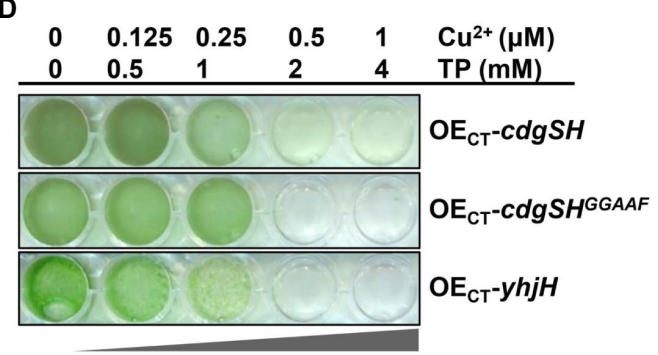

E

\begin{tabular}{llllll}
0 & 0.125 & 0.25 & 0.5 & 1 & $\mathrm{Cu}^{2+}(\mu \mathrm{M})$ \\
0 & 0.5 & 1 & 2 & 4 & $\operatorname{TP}(\mathrm{mM})$ \\
\hline
\end{tabular}

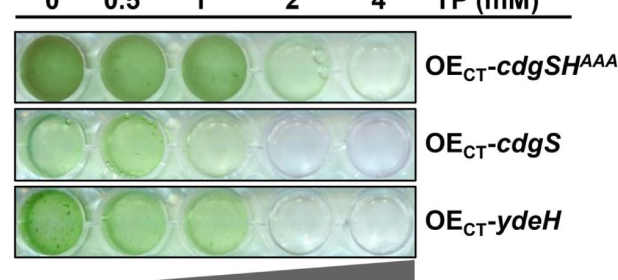

F

\begin{tabular}{llllll}
0 & 0.125 & 0.25 & 0.5 & 1 & $\mathrm{Cu}^{2+}(\mu \mathrm{M})$ \\
0 & 0.5 & 1 & 2 & 4 & $\mathrm{TP}(\mathrm{mM})$ \\
\hline
\end{tabular}

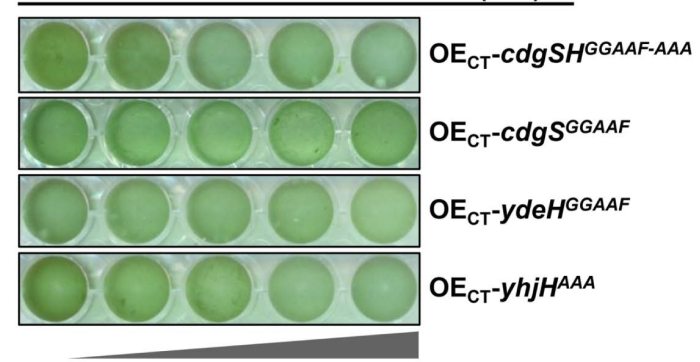

FIGURE 5 | High levels ectopic inductions of PDE or DGC activities inhibited heterocyst development. (A-C) Heterocyst frequency of the indicated overexpression strains at $24 \mathrm{~h}$ after nitrogen stepdown with five concentrations of inducers. The black triangle indicated the increasing levels of the inducers added to the culture media (from left to right column): $0 \mu \mathrm{M}$ copper and $0 \mathrm{mM}$ theophylline, $0.125 \mu \mathrm{M}$ copper and $0.5 \mathrm{mM}$ theophylline, $0.25 \mu \mathrm{M}$ copper and $1 \mathrm{mM}$ theophylline, $0.5 \mu \mathrm{M}$ copper and $2 \mathrm{mM}$ theophylline, and $1 \mu \mathrm{M}$ copper and $4 \mathrm{mM}$ theophylline. (A) The ectopic overexpression of enzymes with PDE activity, including Anabaena CdgSH (strain $\mathrm{OE}_{\mathrm{CT}} \mathrm{CdgSH}$ ), CdgSH variants that abolished the DGC activity (strain $\mathrm{OE}_{\mathrm{CT}}{ }^{-C d g S H^{G G A A F}}$ ) and YhjH from E. coli (strain OE ${ }_{\mathrm{CT}}^{-}$yhjH). (B) The overexpression

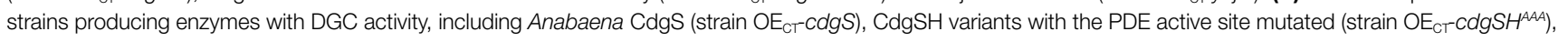
and $\mathrm{YdeH}$ from E. coli (strain $\mathrm{OE}_{\mathrm{CT}^{-}} y d e H$ ). (C) The overexpression strains with production of enzymes that have no active PDE and/or DGC activity, including the

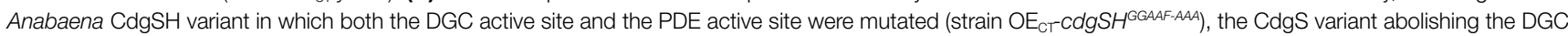

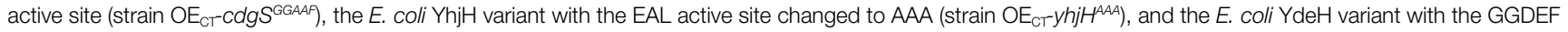
active site changed to GGAAF (strain $\mathrm{OE}_{\mathrm{CT}}-y d \mathrm{H}^{\mathrm{GGAAF}}$ ). (D-F) The growth of different strains as in (A-C) tested in 24-well plates. All cultures were started with a similar OD at 0.3 diluted from a pre-culture and imaged after 4 days of incubation.

We also compared the growth capacity of different strains under diazotrophic conditions. As the proportion of heterocysts decreased, the diazotrophic growth capacity of the strains decreased as well (Figures 5D-F; Supplementary Figure S5B). The growth capacity of control strains remained similar under different inducer conditions. Taken together, our results showed that depending on the promoter used (native promoters of $c d g S H$ or $c d g S$, or the synthetic CT promoter), and the concentrations of the inducers employed, expression of the synthase or hydrolase activity of c-di-GMP may have different outcomes. When expressed under the ectopic promoter (CT promoter) to a high level, both the hydrolase activity and the synthase activity could lead to a repressive effect on heterocyst 
differentiation, suggesting that intracellular c-di-GMP homeostasis in Anabaena is important for heterocyst development.

\section{DISCUSSION}

Heterocyst differentiation in cyanobacteria is regulated by multiple signals (Zhang et al., 2006). In this study, we provide evidence that the second messenger c-di-GMP constitutes a new signal for heterocyst differentiation, as already suggested by a previous study (Neunuebel and Golden, 2008). First, the level of c-di-GMP increases transiently following the initiation of heterocyst differentiation by deprivation of combined nitrogen (Figure 1). This accumulation profile is similar to, but lags behind, that of 2-oxoglutarate, a trigger of heterocyst formation (Laurent et al., 2005). It has been proposed that CdgS acts upstream of HetR, the master regulator of heterocyst development, and is required for the up regulation of pats encoding an inhibitor of heterocyst differentiation (Huang et al., 2004). The time window where the dynamic changes in the level of c-di-GMP was observed in this study is consistent with the early action of this second messenger in heterocyst development.

Secondly, when the c-di-GMP levels are modified by ectopic expression of two well-characterized genes from $\mathrm{E}$. coli, $y \mathrm{deH}$, and $y h j H$, which could synthesize or hydrolyze c-di-GMP, respectively (Pesavento et al., 2008), heterocyst formation was inhibited (Figure 5). Therefore, the regulated change in the intracellular levels of c-di-GMP, and thus its homeostasis, is critical for heterocyst formation. However, when each of the genes involved in c-di-GMP metabolism was inactivated, none of the mutants produced such a strong phenotype. Genetic analysis of the genes revealed that at the single gene level, the role of c-di-GMP signal in heterocyst development was mostly attributed to the functions of the c-di-GMP synthetase CdgS and c-di-GMP hydrolase CdgSH (Figures 3, 4; Tables 1 and 2). The phenotypes of the two-corresponding mutant $\Delta c d g S H$ and $\Delta c d g S$ indicated the c-di-GMP regulates heterocyst frequency at different stages of the developmental process (Figure 2). Therefore, it is likely that at least some of the 16 genes encoding c-di-GMP synthesis or hydrolysis activities may have redundant functions in regulating heterocyst frequency. Such genetic redundancy may also partly account for the dynamic changes in the levels of c-di-GMP during heterocyst development, which cannot be fully explained by the action of CdgS and $\mathrm{CdgSH}$ alone. Indeed, following combined-nitrogen deprivation, it takes about $3 \mathrm{~h}$ for c-di-GMP to climb to the peak level, then to decrease gradually to the basal level (Figure 1). At the transcriptional level, the amount of $c d g S$ transcript detected (encoding a c-di-GMP synthesis enzyme) remains stable at $3 \mathrm{~h}$ post induction, but that of $c d g S H$ (encoding a dominant hydrolase activity of c-di-GMP) decreases (Figure 2). The combined effect of their expression is consistent with the increase in c-di-GMP level observed at the same time point. However, after $3 \mathrm{~h}$, the expression level of $c d g S$ starts to increase, while that of $c d g S H$ remains at a low level (Figure 2), which together should lead to an overall increase in c-di-GMP levels rather than a decrease as shown in Figure 1. Thus, although our genetic data indicate that $\mathrm{CdgSH}$ and $\mathrm{CdgS}$ appear to be the major individual players in regulating heterocyst frequency, other c-di-GMP metabolic enzymes must play important roles as well. Mutant strains, in which all c-di-GMP synthesis or degradation activities could be removed, respectively, would help us to elucidate the essential function of c-di-GMP in regulating heterocyst formation. In addition, an assessment of cell-type specific transcription of genes encoding all c-di-GMP metabolic enzymes would be necessary for a better understanding of their function in heterocyst development. In addition, as discussed below, these enzymes are also regulated at the enzymatic level too, which may also contribute to the dynamic changes of c-di-GMP under different environmental conditions.

Previously, systematic inactivation of 14 genes involved in c-di-GMP turnover was reported, which led to the identification of $c d g S$. In this study, systematic markerless deletion of all 16 genes by using the genome editing technique based on CRISPR-Cpf1 led to the identification of a new gene, $c d g S H$, required for heterocyst frequency (Neunuebel and Golden, 2008). The CRISPR-Cpf1 system allowed us to delete with efficiency a gene from a chromosome without leaving an antibiotic-resistance marker, so that the phenotype of the mutant could be analyzed without adding any antibiotic in the growth medium. The presence of a selective agent may affect phenotypic analysis, in particular when the phenotype is weak, such as that exhibited by the $c d g S H$ mutant. Markerless deletion also simplified the deletion of multiple genes from the chromosome one after another with no limitation imposed by the use of selective antibiotics, and it could in addition avoid the polar effect caused to the downstream genes (Niu et al., 2019).

The C-terminal region of $\mathrm{CdgSH}$ has both a GGDEF and an EAL domain. Consistent with its domain structure, our biochemical data in vitro shows that it has the cyclase activity for c-di-GMP synthesis as well as the hydrolase activity for c-di-GMP degradation (Figure 3). Although the substrate binding efficiencies for both domains are similar, the catalytic activity of the hydrolase domain is more efficient than that of the cyclase domain, leading to c-di-GMP hydrolysis as the net outcome of the $\mathrm{CdgSH}$ activity in vitro. In addition, a feedback inhibition of c-di-GMP synthetase, a characteristic of all c-di-GMP cyclase with the presence the auto inhibition site (Chan et al., 2004; Wassmann et al., 2007), also revealed in $\mathrm{CdgSH}$. The $\mathrm{N}$ terminal part of $\mathrm{CdgSH}$ possesses three sensor domains (FHA, PAS, and GAF); therefore, c-di-GMP turnover due to the activities of $\mathrm{CdgSH}$ should be highly regulated by different input signals acting on these sensor domains. Many PAS and GAF domains in bacteria are light or oxygen sensors due to their binding capacity of pigments, such as flavin mononucleotide, heme, phycocyanobilin, biliverdin, and so on (Christie et al., 1999; Gilles-Gonzalez and Gonzalez, 2004; Savakis et al., 2012; Enomoto et al., 2014; Narikawa et al., 2015). However, the PAS and GAF domains in CdgSH did not contain those conserved residues for pigment binding. Therefore, the signals that regulate the activities of $\mathrm{CdgSH}$ remain to be uncovered. In comparison, $\mathrm{CdgS}$ functions differently since it has only the GGDEF domain and thus the cyclase activity for c-di-GMP synthesis. CdgS has a putative response regulator domain and may also be controlled by signals that need to be identified (Supplementary Figure S1). 
Overall, our genetic studies of the two genes in c-di-GMP metabolism, and the biochemical characterization of the corresponding enzymes, together with the use of heterologous enzymes from $E$. coli, all point out that the function of the two proteins in regulating heterocyst frequency is mediated by their enzymatic activity in c-di-GMP turnover. These results pave the way for further understanding the signaling function of c-di-GMP in Anabaena. It should be noted that although Anabaena has a large number of genes encoding proteins for c-di-GMP synthesis or degradation, no c-di-GMP receptors based on sequence similarity to those already known in other bacteria have been identified in this organism. Therefore, c-di-GMP signaling in cyanobacteria must have distinct features, and their identification will be a key for our understanding the function of c-di-GMP in the regulation of cyanobacterial physiology.

\section{DATA AVAILABILITY STATEMENT}

The original contributions presented in the study are included in the article/Supplementary Material, further inquiries can be directed to the corresponding author.

\section{AUTHOR CONTRIBUTIONS}

$\mathrm{C}-\mathrm{CZ}, \mathrm{XZ}$, and J-YZ designed the research. MH performed the research. $\mathrm{MH}, \mathrm{XZ}$, and $\mathrm{C}-\mathrm{CZ}$ analyzed the data and wrote

\section{REFERENCES}

Agostoni, M., Koestler, B. J., Waters, C. M., Williams, B. L., and Montgomery, B. L. (2013). Occurrence of cyclic di-GMP-modulating output domains in cyanobacteria: an illuminating perspective. $m$ Bio 4, e00451-e00413. doi: 10.1128/mBio.00451-13

Barends, T. R. M., Hartmann, E., Griese, J. J., Beitlich, T., Kirienko, N. V., Ryjenkov, D. A., et al. (2009). Structure and mechanism of a bacterial light-regulated cyclic nucleotide phosphodiesterase. Nature 459, 1015-1018. doi: 10.1038/nature07966

Boehm, A., Kaiser, M., Li, H., Spangler, C., Kasper, C. A., Ackermann, M., et al. (2010). Second messenger-mediated adjustment of bacterial swimming velocity. Cell 141, 107-116. doi: 10.1016/j.cell.2010.01.018

Burdette, D. L., Monroe, K. M., Sotelo-Troha, K., Iwig, J. S., Eckert, B., Hyodo, M., et al. (2011). STING is a direct innate immune sensor of cyclic di-GMP. Nature 478, 515-518. doi: 10.1038/nature10429

Callahan, S. M., and Buikema, W. J. (2001). The role of HetN in maintenance of the heterocyst pattern in Anabaena sp PCC 7120. Mol. Microbiol. 40, 941-950. doi: 10.1046/j.1365-2958.2001.02437.x

Chan, C., Paul, R., Samoray, D., Amiot, N. C., Giese, B., Jenal, U., et al. (2004). Structural basis of activity and allosteric control of diguanylate cyclase. Proc. Natl. Acad. Sci. U. S. A. 101, 17084-17089. doi: 10.1073/pnas.0406134101

Chou, S. H., and Galperin, M. Y. (2016). Diversity of cyclic di-GMP-binding proteins and mechanisms. J. Bacteriol. 198, 32-46. doi: 10.1128/jb.00333-15

Christen, B., Christen, M., Paul, R., Schmid, F., Folcher, M., Jenoe, P., et al. (2006). Allosteric control of cyclic di-GMP signaling. J. Biol. Chem. 281, 32015-32024. doi: 10.1074/jbc.M603589200

Christie, J. M., Salomon, M., Nozue, K., Wada, M., and Briggs, W. R. (1999). LOV (light, oxygen, or voltage) domains of the blue-light photoreceptor phototropin (nph1): binding sites for the chromophore flavin mononucleotide. Proc. Natl. Acad. Sci. U. S. A. 96, 8779-8783. doi: 10.1073/pnas.96.15.8779

Del Medico, L., Cerletti, D., Schachle, P., Christen, M., and Christen, B. (2020). The type IV pilin PilA couples surface attachment and cell-cycle initiation the manuscript. All authors contributed to the article and approved the submitted version.

\section{FUNDING}

This work was supported by Youth Program of National Natural Science Foundation of China (Grant No. 31800033), National Natural Science Foundation of China (Grant No. 92051106), the Key Research Program of Frontier Sciences of the Chinese Academy of Sciences (Grant No. QYZDJ-SSW-SMC016), and the Featured Institute Service Projects from the Institute of Hydrobiology, the Chinese Academy of Sciences (Grant Nos. Y85Z061601 and Y65Z021501).

\section{ACKNOWLEDGMENTS}

We would like to thank Min Wang from the Analysis and Testing Center of Institute of Hydrobiology, Chinese Academy of Sciences for support in the measurement using HPLC and UPLC-MS/MS.

\section{SUPPLEMENTARY MATERIAL}

The Supplementary Material for this article can be found online at: https://www.frontiersin.org/articles/10.3389/fmicb.2021.793336/ full\#supplementary-material

in Caulobacter crescentus. Proc. Natl. Acad. Sci. U. S. A. 117, 9546-9553. doi: $10.1073 /$ pnas.1920143117

Elhai, J., and Khudyakov, I. (2018). Ancient association of cyanobacterial multicellularity with the regulator HetR and an RGSGR pentapeptide-containing protein (PatX). Mol. Microbiol. 110, 931-954. doi: 10.1111/mmi.14003

ElhaI, J., Vepritskiy, A., MuroPastor, A. M., Flores, E., and Wolk, C. P. (1997). Reduction of conjugal transfer efficiency by three restriction activities of Anabaena sp. strain PCC 7120. J. Bacteriol. 179, 1998-2005. doi: 10.1128/ jb.179.6.1998-2005.1997

Enomoto, G., Ni Ni, W., Narikawa, R., and Ikeuchi, M. (2015). Three cyanobacteriochromes work together to form a light color-sensitive input system for c-di-GMP signaling of cell aggregation. Proc. Natl. Acad. Sci. U. S. A. 112, 8082-8087. doi: 10.1073/pnas.1504228112

Enomoto, G., Nomura, R., Shimada, T., Ni Ni, W., Narikawa, R., and Ikeuchi, M. (2014). Cyanobacteriochrome SesA is a diguanylate cyclase that induces cell aggregation in Thermosynechococcus. J. Biol. Chem. 289, 24801-24809. doi: $10.1074 /$ jbc.M114.583674

Gallagher, K. A., Schumacher, M. A., Bush, M. J., Bibb, M. J., Chandra, G., Holmes, N. A., et al. (2020). c-di-GMP arms an anti-sigma to control progression of multicellular differentiation in Streptomyces. Mol. Cell 77, 586-599.e6. doi: 10.1016/j.molcel.2019.11.006

Gilles-Gonzalez, M. A., and Gonzalez, G. (2004). Signal transduction by hemecontaining PAS-domain proteins. J. Appl. Physiol. 96, 774-783. doi: 10.1152/ japplphysiol.00941.2003

Golden, J. W., Whorff, L. L., and Wiest, D. R. (1991). Independent regulation of nifhdk operon transcription and dna rearrangement during heterocyst differentiation in the cyanobacterium anabaena sp strain PCC 7120. J. Bacteriol. 173, 7098-7105. doi: 10.1128/jb.173.22.7098-7105.1991

Hengge, R. (2009). Principles of c-di-GMP signalling in bacteria. Nat. Rev. Microbiol. 7, 263-273. doi: 10.1038/nrmicro2109

Herrero, A., and Flores, E. (2019). Genetic responses to carbon and nitrogen availability in Anabaena. Environ. Microbiol. 21, 1-17. doi: 10.1111/1462-2920.14370 
Hickman, J. W., and Harwood, C. S. (2008). Identification of FleQ from Pseudomonas aeruginosa as a c-di-GMP-responsive transcription factor. Mol. Microbiol. 69, 376-389. doi: 10.1111/j.1365-2958.2008.06281.x

Huang, X., Dong, Y. Q., and Zhao, J. D. (2004). HetR homodimer is a DNAbinding protein required for heterocyst differentiation, and the DNA-binding activity is inhibited by PatS. Proc. Natl. Acad. Sci. U. S. A. 101, 4848-4853. doi: 10.1073 pnas. 0400429101

Hug, I., Deshpande, S., Sprecher, K. S., Pfohl, T., and Jenal, U. (2017). Second messenger-mediated tactile response by a bacterial rotary motor. Science 358, 531-534. doi: 10.1126/science.aan5353

Kulesekara, H., Lee, V., Brencic, A., Liberati, N., Urbach, J., Miyata, S., et al. (2006). Analysis of Pseudomonas aeruginosa diguanylate cyclases and phosphodiesterases reveals a role for bis-(3'-5')-cyclic-GMP in virulence. Proc. Natl. Acad. Sci. U. S. A. 103, 2839-2844. doi: 10.1073/pnas.0511090103

Kumar, K., Mella-Herrera, R. A., and Golden, J. W. (2010). Cyanobacterial heterocysts. Cold Spring Harb. Perspect. Biol. 2:19. doi: 10.1101/cshperspect. a000315

Laurent, S., Chen, H., Bedu, S., Ziarelli, F., Peng, L., and Zhang, C. C. (2005). Nonmetabolizable analogue of 2-oxoglutarate elicits heterocyst differentiation under repressive conditions in Anabaena sp. PCC 7120. Proc. Natl. Acad. Sci. U. S. A. 102, 9907-9912. doi: 10.1073/pnas.0502337102

Li, W. H., Li, M., Hu, L. H., Zhu, J. P., Xie, Z. W., Chen, J. R., et al. (2018). HpoR, a novel c-di-GMP effective transcription factor, links the second messenger's regulatory function to the mycobacterial antioxidant defense. Nucleic Acids Res. 46, 3595-3611. doi: 10.1093/nar/gky146

Lori, C., Ozaki, S., Steiner, S., Bohm, R., Abel, S., Dubey, B. N., et al. (2015). Cyclic di-GMP acts as a cell cycle oscillator to drive chromosome replication. Nature 523, 236-239. doi: 10.1038/nature14473

Martin-Moldes, Z., Blazquez, B., Baraquet, C., Harwood, C. S., Zamarro, M. T., and Diaz, E. (2016). Degradation of cyclic diguanosine monophosphate by a hybrid two-component protein protects Azoarcus sp strain CIB from toluene toxicity. Proc. Natl. Acad. Sci. U. S. A. 113, 13174-13179. doi: 10.1073/ pnas. 1615981113

Muro-Pastor, A. M., and Hess, W. R. (2012). Heterocyst differentiation: from single mutants to global approaches. Trends Microbiol. 20, 548-557. doi: 10.1016/j.tim.2012.07.005

Narikawa, R., Nakajima, T., Aono, Y., Fushimi, K., Enomoto, G., Ni Ni, W., et al. (2015). A biliverdin-binding cyanobacteriochrome from the chlorophyll d-bearing cyanobacterium Acaryochloris marina. Sci. Rep. 5:7950. doi: 10.1038/ srep07950

Neunuebel, M. R., and Golden, J. W. (2008). The Anabaena sp strain PCC 7120 gene all2874 encodes a diguanylate cyclase and is required for normal heterocyst development under high-light growth conditions. J. Bacteriol. 190, 6829-6836. doi: 10.1128/jb.00701-08

Niu, T. C., Lin, G. M., Xie, L. R., Wang, Z. Q., Xing, W. Y., Zhang, J. Y., et al. (2019). Expanding the potential of CRISPR-cpf1-based genome editing technology in the cyanobacterium Anabaena PCC 7120. ACS Synth. Biol. 8, 170-180. doi: 10.1021/acssynbio.8b00437

Paul, R., Abel, S., Wassmann, P., Beck, A., Heerklotz, H., and Jenal, U. (2007). Activation of the diguanylate cyclase $\mathrm{PleD}$ by phosphorylation-mediated dimerization. J. Biol. Chem. 282, 29170-29177. doi: 10.1074/jbc.M704702200

Paul, R., Weiser, S., Amiot, N. C., Chan, C., Schirmer, T., Giese, B., et al. (2004). Cell cycle-dependent dynamic localization of a bacterial response regulator with a novel di-guanylate cyclase output domain. Genes Dev. 18, 715-727. doi: 10.1101/gad.289504

Pesavento, C., Becker, G., Sommerfeldt, N., Possling, A., Tschowri, N., Mehlis, A., et al. (2008). Inverse regulatory coordination of motility and curli-mediated adhesion in Escherichia coli. Genes Dev. 22, 2434-2446. doi: 10.1101/ gad. 475808

Romling, U., Galperin, M. Y., and Gomelsky, M. (2013). Cyclic di-GMP: the first 25 years of a universal bacterial second messenger. Microbiol. Mol. Biol. Rev. 77, 1-52. doi: 10.1128/mmbr.00043-12

Ross, P., Weinhouse, H., Aloni, Y., Michaeli, D., Weinbergerohana, P., Mayer, R., et al. (1987). Regulation of cellulose synthesis in Acetobacter-xylinum by cyclic diguanylic acid. Nature 325, 279-281. doi: 10.1038/325279a0

Savakis, P., De Causmaecker, S., Angerer, V., Ruppert, U., Anders, K., Essen, L. O., et al. (2012). Light-induced alteration of c-di-GMP level controls motility of Synechocystis sp PCC 6803. Mol. Microbiol. 85, 239-251. doi: 10.1111/j. 1365-2958.2012.08106.x
Schmidt, A. J., Ryjenkov, D. A., and Gomelsky, M. (2005). The ubiquitous protein domain EAL is a cyclic diguanylate-specific phosphodiesterase: enzymatically active and inactive EAL domains. J. Bacteriol. 187, 4774-4781. doi: $10.1128 / \mathrm{jb} .187 .14 .4774-4781.2005$

Snyder, R. A., Ellison, C. K., Severin, G. B., Whitfield, G. B., Waters, C. M., and Brun, Y. V. (2020). Surface sensing stimulates cellular differentiation in Caulobacter crescentus. Proc. Natl. Acad. Sci. U. S. A. 117, 17984-17991. doi: 10.1073/pnas.1920291117

Stanier, R. Y., Kunisawa, R., Mandel, M., and Cohen-Bazire, G. (1971). Purification and properties of unicellular blue-green algae (order Chroococcales). Bacteriol. Rev. 35, 171-205. doi: 10.1128/mmbr.35.2.171-205.1971

Tchigvintsev, A., Xu, X., Singer, A., Chang, C., Brown, G., Proudfoott, M., et al. (2010). Structural insight into the mechanism of c-di-GMP hydrolysis by EAL domain phosphodiesterases. J. Mol. Biol. 402, 524-538. doi: 10.1016/j. jmb.2010.07.050

Tischler, A. D., and Camilli, A. (2004). Cyclic diguanylate (c-di-GMP) regulates Vibrio cholerae biofilm formation. Mol. Microbiol. 53, 857-869. doi: 10.1111/j. 1365-2958.2004.04155.x

Tschowri, N., Schumacher, M. A., Schlimpert, S., Chinnam, N. B., Findlay, K. C., Brennan, R. G., et al. (2014). Tetrameric c-di-GMP mediates effective transcription factor dimerization to control Streptomyces development. Cell 158, 1136-1147. doi: 10.1016/j.cell.2014.07.022

Tuckerman, J. R., Gonzalez, G., Sousa, E. H. S., Wan, X., Saito, J. A., Alam, M., et al. (2009). An oxygen-sensing diguanylate cyclase and phosphodiesterase couple for c-di-GMP control. Biochemistry 48, 9764-9774. doi: 10.1021/bi901409g

Wassmann, P., Chan, C., Paul, R., Beck, A., Heerklotz, H., Jenal, U., et al. (2007). Structure of $\mathrm{BeF}_{3}-$-modified response regulator PleD: implications for diguanylate cyclase activation, catalysis, and feedback inhibition. Structure 15, 915-927. doi: 10.1016/j.str.2007.06.016

Wolk, C. P., Ernst, A., and Elhai, J. (1994). "Heterocyst metabolism and development," in The Molecular Biology of Cyanobacteria. ed. D. A. Bryant (Dordrecht: Kluwer Academic Publishers), 769-823.

Xing, W. Y., Xie, L. R., Zeng, X. L., Yang, Y. L., and Zhang, C. C. (2020). Functional dissection of genes encoding dna polymerases based on conditional mutants in the heterocyst-forming cyanobacterium Anabaena PCC 7120 Front. Microbiol. 11:1108. doi: 10.3389/fmicb.2020.01108

Xu, Z. W., Zhang, H. N., Zhang, X. R., Jiang, H. W., Liu, C. X., Wu, F. L., et al. (2019). Interplay between the bacterial protein deacetylase CobB and the second messenger c-di-GMP. EMBO J. 38:e100948. doi: 10.15252/embj.2018100948

Yang, C. X., Cui, C. Y., Ye, Q. M., Kan, J. H., Fu, S. N., Song, S. H., et al. (2017). Burkholderia cenocepacia integrates cis-2-dodecenoic acid and cyclic dimeric guanosine monophosphate signals to control virulence. Proc. Natl. Acad. Sci. U. S. A. 114, 13006-13011. doi: 10.1073/pnas.1709048114

Yoon, H. S., and Golden, J. W. (1998). Heterocyst pattern formation controlled by a diffusible peptide. Science 282, 935-938. doi: 10.1126/science.282.5390.935

Zhang, C. C., Laurent, S., Sakr, S., Peng, L., and Bedu, S. (2006). Heterocyst differentiation and pattern formation in cyanobacteria: a chorus of signals. Mol. Microbiol. 59, 367-375. doi: 10.1111/j.1365-2958.2005.04979.x

Zhang, S.-R., Lin, G.-M., Chen, W.-L., Wang, L., and Zhang, C.-C. (2013). ppGpp metabolism is involved in heterocyst development in the cyanobacterium anabaena sp strain PCC 7120. J. Bacteriol. 195, 4536-4544. doi: 10.1128/jb.00724-13

Conflict of Interest: The authors declare that the research was conducted in the absence of any commercial or financial relationships that could be construed as a potential conflict of interest.

Publisher's Note: All claims expressed in this article are solely those of the authors and do not necessarily represent those of their affiliated organizations, or those of the publisher, the editors and the reviewers. Any product that may be evaluated in this article, or claim that may be made by its manufacturer, is not guaranteed or endorsed by the publisher.

Copyright $\odot 2021$ Huang, Zhang, Zeng and Zhang. This is an open-access article distributed under the terms of the Creative Commons Attribution License (CC BY). The use, distribution or reproduction in other forums is permitted, provided the original author(s) and the copyright owner(s) are credited and that the original publication in this journal is cited, in accordance with accepted academic practice. No use, distribution or reproduction is permitted which does not comply with these terms. 